IPPP-15-23, DCPT-15-46

\title{
Electroweak Baryogenesis from Exotic Electroweak Symmetry Breaking
}

\author{
Nikita Blinov ${ }^{(a, b)}$, Jonathan Kozaczuk ${ }^{(a)}$, \\ David E. Morrissey ${ }^{(a)}$, and Carlos Tamarit ${ }^{(c)}$ \\ (a) TRIUMF, 4004 Wesbrook Mall, Vancouver, BC V6T 2A3, Canada \\ (b) Department of Physics and Astronomy, University of British Columbia, \\ Vancouver, BC V6T 1Z1, Canada \\ (c) Institute for Particle Physics Phenomenology, Department of Physics \\ Durham University, Durham DH1 3LE, United Kingdom \\ email:nblinov@triumf.ca,jkozaczuk@triumf.ca, \\ dmorri@triumf.ca,carlos.tamarit@durham.ac.uk
}

August 20, 2015

\begin{abstract}
We investigate scenarios in which electroweak baryogenesis can occur during an exotic stage of electroweak symmetry breaking in the early Universe. This transition is driven by the expectation value of a new electroweak scalar instead of the standard Higgs field. A later, second transition then takes the system to the usual electroweak minimum, dominated by the Higgs, while preserving the baryon asymmetry created in the first transition. We discuss the general requirements for such a two-stage electroweak transition to be suitable for electroweak baryogenesis and present a toy model that illustrates the necessary ingredients. We then apply these results to construct an explicit realization of this scenario within the inert two Higgs doublet model. Despite decoupling the Higgs from the symmetry-breaking transition required for electroweak baryogenesis, we find that this picture generically predicts new light states that are accessible experimentally.
\end{abstract}




\section{Introduction}

Electroweak baryogenesis (EWBG) is an elegant mechanism for the generation of the baryon asymmetry during the electroweak phase transition [1 3$]$. If this phase transition is strongly first order, it proceeds through the formation of bubbles of broken electroweak phase that expand rapidly within the surrounding region of symmetric phase. This departure from thermodynamic equilibrium, together with $C$ (charge) and $C P$ (charge-parity) violation in particle scattering at the bubble walls and $B+L$ violation by electroweak sphaleron transitions, satisfy the necessary Sakharov conditions for baryogenesis [4].

The standard realization of EWBG is driven by the Standard Model (SM) Higgs field (or a Higgs sector from which the SM-like Higgs emerges). At high temperatures, the Higgs is stabilized at the symmetry-preserving origin of its potential by thermal effects. As the temperature falls, the Higgs field develops a vacuum expectation value (VEV) and breaks the manifest electroweak invariance. EWBG requires that this transition be strongly first-order, both to generate bubble walls near which efficient baryon production can take place and to suppress $B+L$ violation by sphaleron transitions within the broken-phase bubbles. The latter condition is more severe, and is usually quoted as

$$
\frac{v_{c}}{T_{c}} \gtrsim 1
$$

where $v_{c}$ is the Higgs VEV at the critical temperature $T_{c}$ below which the first-order phase transition can occur ${ }^{1}$ Violation of $C$ and $C P$ in scattering with the Higgs bubble wall also emerges from interactions with the changing Higgs field.

This baryogenesis mechanism is particularly intriguing because the dynamics takes place near the weak scale, suggesting that it can be probed experimentally with current or upcoming data. Indeed, EWBG has already been tested and ruled out within the Standard Model (SM), which contains all the necessary ingredients. The are two reasons for this: the electroweak phase transition (EWPT) is not first order for the observed Higgs boson mass $m_{h} \simeq 125 \mathrm{GeV}$ [7,8], and the measured $C P$ violation from the Cabbibo-Kobayashi-Maskawa matrix is insufficient 911 .

Electroweak baryogenesis is able to generate the observed baryon asymmetry in a number of extensions of the SM. However, most of these realizations are becoming very strongly constrained as well. To drive the EWPT to be strongly first order, the new physics must couple significantly to the SM Higgs, and this can lead to modifications to Higgs boson production and decay rates. For example, the EWPT can be made strong in supersymmetric extensions of the SM through the effects of a light scalar top (stop) superpartner [12, 13, by mixing with a new singlet scalar 14 16], or even by arranging a tree-level barrier between the

\footnotetext{
1 This condition is gauge dependent and has significant theoretical uncertainties 5 but is often a good rule of thumb [6]. We discuss gauge dependence and other uncertainties in more detail in Section 3.3
} 
Higgs vacuum and the origin. Comparing to data, the stop-driven scenario is mostly ruledout by precision measurements of the Higgs production and decay rates at the LHC [17 20] as well as by direct stop searches [21,22], while Higgs measurements also limit the singletdriven scenario to a small subset of the model parameter space [23,24]. Coleman-Weinberglike scenarios with tree-level barriers involve heavier exotic fields, but could be probed by precise measurements of the Higgs couplings [25, 26]. Similar conclusions are found in other extensions of the SM that produce a strongly first-order EWPT [27].

New sources of $C P$ violation are also needed for viable EWBG, and these must connect to the SM Higgs so that they can be enhanced by the advancing bubble walls. This will typically lead to new contributions to the permanent electric dipole moments (EDMs) of leptons and baryons at the two-loop level [28]. In supersymmetric extensions, the main new source of $C P$ violation in EWBG typically comes from the charginos and neutralinos, and their contribution to EDMs is larger than the current experimental limits except in a small resonantly enhanced window [29]. Similar bounds are found in other theories [30].

In this work, we investigate a non-standard realization of electroweak baryogenesis involving a two-stage phase transition as a means to sidestep some of these constraints, making use of the mechanism first introduced in Ref. [31. The key idea is to have an initial stage of electroweak symmetry breaking induced by the VEV of an exotic $S U(2)_{L}$-charged scalar rather than the standard Higgs. At some later time, the system will evolve from the exotic vacuum to the standard Higgs vacuum that we occupy today. Baryon production via EWBG could then occur in the first, exotic field transition. As long as the transition to the standard Higgs vacuum maintains enough electroweak symmetry breaking (or if it is strongly firstorder), the baryons created in the initial phase transition will be preserved. Additional new physics (beyond the exotic scalar) needed to drive the strong initial transition or to generate $C P$ violation can couple primarily to the exotic scalar rather than the SM-like Higgs field. This suggests that the bounds on EWBG can be relaxed in this scenario. We will see that this is only partially true.

Multi-stage phase transitions have been considered before in the context of EWBG. In scenarios with a singlet scalar that helps to drive a strongly first-order Higgs phase transition, the singlet is often found to develop a VEV before the Higgs field [23, 24, 27, 32,34]. Electroweak symmetry breaking by an exotic doublet was suggested in Ref. [35] with the goal of inducing EWBG in a strongly first-order phase transition from the exotic vacuum to the Higgs vacuum. This does not work because of a strong suppression of sphalerons in the initial exotic broken phase 36 .

Two-stage electroweak symmetry breaking with EWBG taking place in the first step was proposed in Ref. [31]. Here, we aim to investigate and extend this mechanism further. Relative to Ref. [31, which was based on a triplet extension of the SM, we elucidate several general criteria for such two-stage electroweak phase transitions, and we exhibit a new realization suitable for EWBG within an inert two-Higgs-doublet model. We also discuss the resulting particle phenomenology of this class of theories. 
The outline of this paper is as follows. In Section 2, we argue that two-stage electroweak phase transitions are difficult to achieve with one field alone, generically requiring additional fields transforming non-trivially under $S U(2)_{L}$. To illustrate what this entails, we present a toy model that realizes a two-stage phase transition in a simple and general way. In Section 3 , we apply these results to a realistic inert two-Higgs-doublet model that exhibits a two-step electroweak symmetry-breaking transition in which the first stage is suitable for EWBG. The experimental constraints on this scenario are studied in Section 4, where we show that an additional contribution to the masses of the exotic scalars is needed, and we exhibit a simple extension of the inert doublet model in which this can occur. Finally, Section 5 is reserved for our conclusions. Some technical details about our treatment of the effective potential and its thermal corrections are collected in a pair of appendices.

\section{Requirements for a Viable Two-Step Transition}

We are interested in scenarios in which electroweak symmetry breaking occurs in two stages in the early Universe, with the first transition proceeding towards an exotic vacuum and the second transition bringing the system (close) to the standard electroweak minimum. In this section we outline the general conditions under which such a two-stage transition can occur in a realistic way, list the additional requirements for successful EWBG, and present a simplified toy model that illustrates what is needed.

\subsection{General Conditions with One Electroweakly Charged Scalar}

A minimal scenario for a two-stage transition would involve a new exotic vacuum in the potential of a single Higgs scalar doublet. At zero temperature the exotic vacuum should lie above the standard Higgs vacuum or disappear entirely, while at high temperature the SM vacuum should be lifted to enable a direct transition from the symmetry-preserving origin to the exotic vacuum. This reversal in the ordering of the free-energies of the vacua implies that the effective potential in the region of the standard vacuum needs to receive stronger thermal corrections than the potential in the region of the exotic minimum. We do not expect this to occur if the false vacuum has a larger VEV than the SM minimum, since thermal corrections to the potential increase for growing values of the fields, until they become roughly constant due to Boltzmann suppression:2 If, on the other hand, the new vacuum lies closer to the origin - which is difficult to realize in a perturbative setting - it would be challenging to obtain $v_{c} / T_{c} \gtrsim 1$ with both a smaller VEV and a higher critical temperature than the ones corresponding to the standard Higgs vacuum. For these reasons we will not consider this possibility further.

\footnotetext{
${ }^{2}$ The growth of thermal corrections for small enough values of the fields can be seen by noting that the leading thermal corrections to the effective potential are given by positive temperature-dependent mass terms, quadratic in the fields.
} 

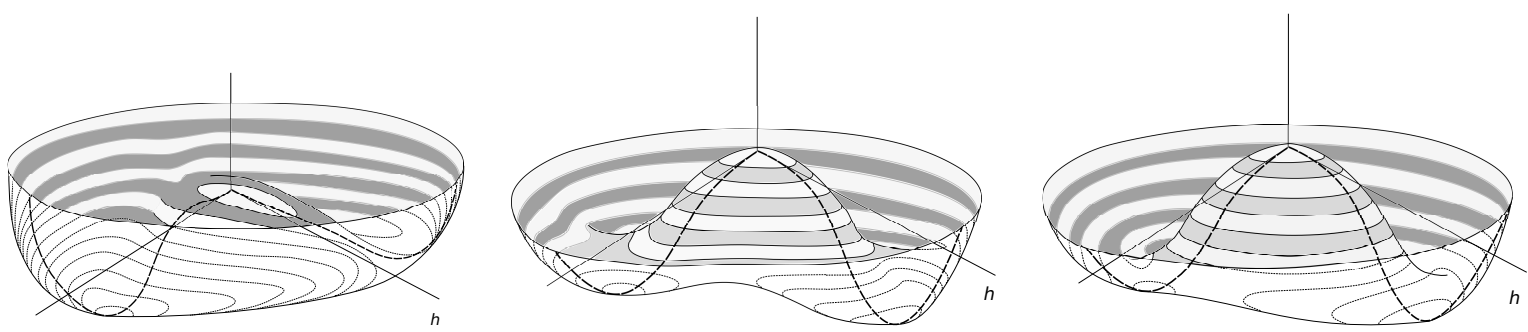

Figure 1: Scenarios with vacua aligned along different field directions. Left: High temperature potential with no vacuum in the Higgs direction, enabling a first-order transition to the exotic vacuum. Middle and Right: Possible low or zero temperature potentials, for which first- (middle) or second-order (right) transitions towards the Higgs vacuum are possible.

\subsection{General Conditions with Two Electroweakly Charged Scalars}

For the remainder of this work we will focus on theories with two electroweakly-charged scalars $\Phi$ and $H$, with $\Phi$ mostly inert and $H$ associated with the standard electroweak vacuum, gauge boson and fermion masses, and the Higgs boson. At zero temperature, $T=0$, electroweak symmetry breaking should therefore be dominated by the VEV of $H$. A VEV for $\Phi$ is also possible, but it must be much smaller than the VEV of $H$ to avoid large corrections to electroweak observables or Higgs boson production rates [37,38]. We will focus on the case where $\langle\Phi\rangle$ is negligible today. Thus, any non-standard vacua present at $T=0$ should be shallower than the Higgs vacuum, or separated by a large barrier.

At very high temperatures, thermal corrections are expected to drive the ground state of the system to the origin of the $(H, \Phi)$ field space. To realize the two-stage scenario we are interested in, the exotic $\Phi$ field must develop a VEV first. This can occur readily if the $H$ field has larger couplings than $\Phi$ to light matter fields in the cosmological plasma, since these will provide a stronger thermal stabilization of the $H$ direction. If EWBG is to occur in the first transition along the $\Phi$ direction, it must also be strongly first order to suppress non-perturbative baryon washout processes such as sphaleron transitions [39, 40] or electroweak monopoles 31.

As the temperature falls further, the system should evolve from the exotic vacuum to something close to the standard electroweak minimum dominated by the VEV of $H$, as shown in Fig. 1. This later transition can be first- or second-order. However, if it is to preserve the baryon number generated by EWBG in the initial transition, it must satisfy some additional conditions. When the second transition is first-order, corresponding to the middle panel of Fig. 11, it must complete efficiently enough that it does not inject a large amount of entropy that would overly dilute the baryon asymmetry [31]. If the second transition is second-order, as in the right panel of Fig. 1, the total amount of electroweak symmetry breaking must be large enough to suppress baryon washout by sphaleron transitions $[39,40]$. In the case of two 
electroweak doublets, this translates into a condition of the form 41

$$
\frac{\sqrt{\langle H\rangle^{2}+\langle\Phi\rangle^{2}}}{T} \gtrsim 1 / \sqrt{2}
$$

where it should be noted that there is a significant uncertainty due to the gauge dependence of the effective potential and higher-order parts of the sphaleron transition rate [5]. A similar condition applies when the exotic multiplet occupies a higher-dimensional representation of $S U(2)_{L}$ 41.

\subsection{A Two-Stage Toy Model}

The general conditions for a two-stage electroweak transition described above can be understood simply in a toy model containing a pair of complex scalar fields. While this model does not contain any gauge degrees of freedom, and the first transition is usually not first-order, it will serve as a useful reference for studying realistic theories of electroweak symmetry breaking and EWBG below.

The model consists of two complex scalar fields $\Phi_{1}$ and $\Phi_{2}$ and $N$ copies of a "top" fermion $T$ with Lagrangian

$$
\begin{aligned}
-\mathscr{L}= & -\mathscr{L}_{\text {kinetic }} \\
& -\mu_{1}^{2}\left|\Phi_{1}\right|^{2}-\mu_{2}^{2}\left|\Phi_{2}\right|^{2}+\frac{\lambda_{1}}{2}\left|\Phi_{1}\right|^{4}+\frac{\lambda_{2}}{2}\left|\Phi_{2}\right|^{4}+\lambda_{3}\left|\Phi_{1}\right|^{2}\left|\Phi_{2}\right|^{2} \\
& +\left(y_{T} \Phi_{1} \bar{T}_{L} T_{R}+\text { h.c. }\right),
\end{aligned}
$$

with $\mu_{i}^{2}>0$ and $\lambda_{i}>0$. The potential of this theory has two independent $U(1)$ global symmetries under which each of the complex scalars transforms independently. The negative quadratic interactions will induce VEVs for one or both fields. Choosing a vacuum where both VEVs are real, we expand the fields as

$$
\begin{aligned}
& \Phi_{1}=\left(\varphi_{1}+r_{1}+i A_{1}\right) / \sqrt{2} \\
& \Phi_{2}=\left(\varphi_{2}+r_{2}+i A_{2}\right) / \sqrt{2}
\end{aligned}
$$

where $\varphi_{1,2}$ are the (canonically-normalized) background scalar fields entering the effective potential. The tree-level effective potential then becomes

$$
\begin{aligned}
V_{0}\left(\varphi_{1}, \varphi_{2}\right) & =-\frac{1}{2} \mu_{1}^{2} \varphi_{1}^{2}-\frac{1}{2} \mu_{2}^{2} \varphi_{2}^{2}+\frac{\lambda_{1}}{8} \varphi_{1}^{4}+\frac{\lambda_{2}}{8} \varphi_{2}^{4}+\frac{\lambda_{3}}{4} \varphi_{1}^{2} \varphi_{2}^{2} \\
& =-\frac{1}{2}\left(\mu_{1}^{2} \varphi_{1}^{2}+\mu_{2}^{2} \varphi_{2}^{2}\right)+\frac{\lambda_{3}}{8 \mu_{1}^{2} \mu_{2}^{2}}\left(\mu_{1}^{2} \varphi_{1}^{2}+\mu_{2}^{2} \varphi_{2}^{2}\right)^{2}+\frac{\Delta \lambda_{1}}{8} \varphi_{1}^{4}+\frac{\Delta \lambda_{2}}{8} \varphi_{2}^{4}
\end{aligned}
$$

where the second line is a suggestive rewriting of the potential in terms of

$$
\Delta \lambda_{1}=\lambda_{1}-\frac{\mu_{1}^{2}}{\mu_{2}^{2}} \lambda_{3}, \quad \Delta \lambda_{2}=\lambda_{2}-\frac{\mu_{2}^{2}}{\mu_{1}^{2}} \lambda_{3} .
$$


For any values of $\Delta \lambda_{1}$ and $\Delta \lambda_{2}$, the potential always has local extrema at $\left(v_{1}, 0\right)$ and $\left(0, v_{2}\right)$ with $v_{i}^{2}=2 \mu_{i}^{2} / \lambda_{i}$ and $V\left(v_{i}\right)=-\mu_{i}^{4} / 2 \lambda_{i}$. In the special case of $\Delta \lambda_{1}=\Delta \lambda_{2}=0$, these local extrema both coincide with an ellipse of minima in $\varphi_{1}-\varphi_{2}$ plane defined by $\left(\mu_{1}^{2} \varphi_{1}^{2}+\mu_{2}^{2} \varphi_{2}^{2}\right)=2 \mu_{1}^{2} \mu_{2}^{2} / \lambda_{3}$. Turning on small values of $\Delta \lambda_{1}$ and $\Delta \lambda_{2}$, the ellipse is deformed and the potential develops discrete minima. These minima may coincide with the exclusive extrema at $\left(v_{1}, 0\right)$ and $\left(0, v_{2}\right)$, or they may lie in a valley connecting them. Examining the local stability conditions for the exclusive extrema, $\left(v_{1}, 0\right)$ is stable for $\Delta \lambda_{1}<0$ and $\left(0, v_{2}\right)$ is stable for $\Delta \lambda_{2}<0$. This implies three distinct cases (up to the exchange $1 \leftrightarrow 2$ ):

1. $\Delta \lambda_{1}<0, \Delta \lambda_{2}>0$

$\left(v_{1}, 0\right)$ is a minimum and $\left(0, v_{2}\right)$ is unstable, and they are connected by a smoothly decreasing valley.

2. $\Delta \lambda_{\mathbf{1}}<\mathbf{0}, \boldsymbol{\Delta} \lambda_{\mathbf{2}}<\mathbf{0}$

$\left(v_{1}, 0\right)$ and $\left(0, v_{2}\right)$ are both local minima, and there exists a higher saddle-point barrier within the valley connecting them.

3. $\Delta \lambda_{1}>0, \Delta \lambda_{2}>0$

$\left(v_{1}, 0\right)$ and $\left(0, v_{2}\right)$ are both saddle points, and there exists a deeper minimum within the valley connecting them.

We will be primarily interested in Cases 1 and 2; Case 3 is undesirable because both fields would obtain non-negligible VEVs.

At finite temperature $T$, the effective scalar potential will receive thermal corrections. The leading effect at large $T$ is to modify the scalar squared-mass parameters in Eq. (2.5) according to

$$
\mu_{i}^{2} \rightarrow m_{i}^{2}=\mu_{i}^{2}-a_{i} T^{2}
$$

with

$$
a_{1}=\frac{\lambda_{1}}{6}+\frac{\lambda_{3}}{12}+N \frac{y_{T}^{2}}{12}, \quad a_{2}=\frac{\lambda_{2}}{6}+\frac{\lambda_{3}}{12} .
$$

At very high $T$, both $m_{1}^{2}$ and $m_{2}^{2}$ are negative and the only minimum of the potential lies at the origin. As the temperature falls, one of the $m_{i}^{2}$ will become positive and a VEV will develop along the corresponding field direction. For appropriate values of the parameters, this theory may also undergo a second, later transition in which the other field develops a VEV.

To illustrate a two-stage transition, let us take $\Delta \lambda_{1}<0$ and $\Delta \lambda_{2}>0$ (at $T=0$ ) as in Case 1 above, so that the minimum at $T=0$ lies at $\left(v_{1}, 0\right)$. The $\varphi_{1}$ and $\varphi_{2} \operatorname{VEVs}$ are then analogous to the SM-like Higgs and the exotic scalar fields, respectively. For the first transition to occur exclusively in the $\varphi_{2}$ direction, $m_{2}^{2}$ should become positive before $m_{1}^{2}$, which requires

$$
\mu_{2}^{2} / a_{2}>\mu_{1}^{2} / a_{1}
$$




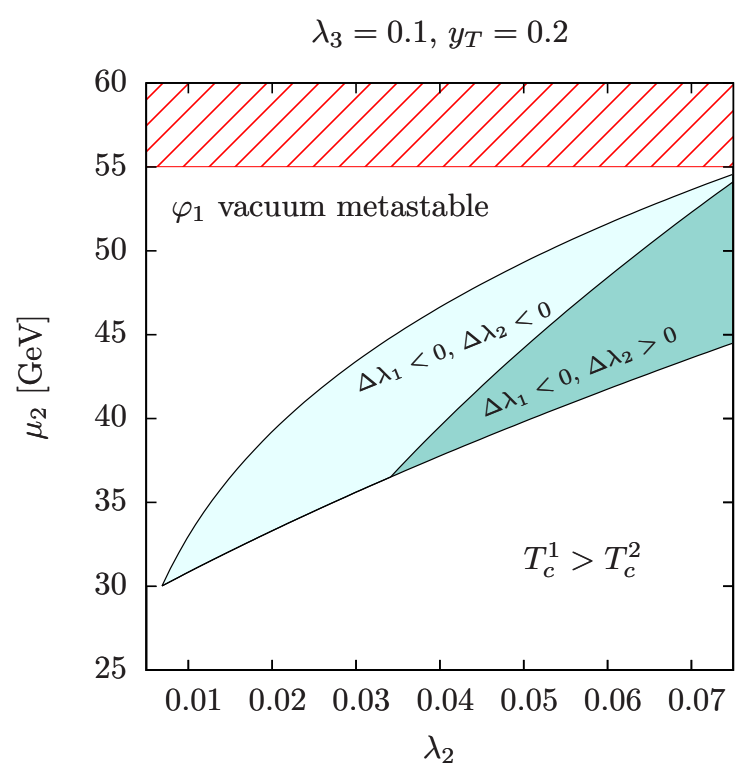

Figure 2: Parameter regions in $\lambda_{2}-\mu_{2}$ plane of the toy model where a two-stage phase transition is likely to occur, first in the "exotic" $\varphi_{2}$ direction and then proceeding to the "standard" $\varphi_{1}$ direction. The regions correspond to the conditions of Eqs. $2.10,2.11,2.12$, 2.13), with $\lambda_{3}=0.1, y_{T}=0.2$ with $N=3$, and $\lambda_{1}$ and $\mu_{1}^{2}$ set as described in the text. All four conditions are satisfied in the darker shaded region.

The inequality can be satisfied if the coupling $y_{T}$ in Eq. $(2.2)$ is large enough or $\lambda_{2}$ is small enough. As the temperature falls further, the minimum in the $\varphi_{2}$ direction will disappear and the field will end up in the $T=0$ minimum at $\left(v_{1}, 0\right)$. This can happen smoothly in a second-order transition, or via tunneling in a first-order transition if a barrier is generated by thermal effects.

A two-step transition can occur in a similar way in Case 2, with $\Delta \lambda_{1}<0$ and $\Delta \lambda_{2}<0$. However, a barrier between the $\varphi_{1}$ and $\varphi_{2}$ vacua is present at tree-level in this case and persists at $T=0$. The second transition is now first order, and there is a danger that it never completes or that it will lead to strong supercooling followed by a substantial injection of entropy. Both outcomes are problematic for electroweak baryogenesis, and we will study them further in the more realistic theories to be considered below.

Many features of this simple toy model will be applicable to extended Higgs sectors giving rise to two-stage transitions where the $\mathrm{VEVs}$ are aligned along one of the field directions at each of the transitions. In particular, we can derive four conditions on the parameter space of the toy model that will serve as a useful guide for realistic theories when we identify $\Phi_{1}$ with the standard Higgs doublet and $\Phi_{2}$ with an exotic electroweak scalar multiplet. Fixing 
$\lambda_{1}, \lambda_{3}$, and $\mu_{1}^{2}$, these conditions can be expressed as inequalities in the $\lambda_{2}-\mu_{2}$ plane:

$$
\begin{array}{ll}
\mu_{2}^{2}<\left(\frac{\lambda_{3}}{\lambda_{1}}\right) \mu_{1}^{2} & \left(\Delta \lambda_{1}<0\right) \\
\mu_{2}^{2}<\sqrt{\frac{\lambda_{2}}{\lambda_{1}}} \mu_{1}^{2} & \left(V\left(v_{1}\right)<V\left(v_{2}\right)\right) \\
\mu_{2}^{2}>\left(\frac{a_{2}}{a_{1}}\right) \mu_{1}^{2} & \left(T_{c}^{2}>T_{c}^{1}\right) \\
\mu_{2}^{2}<\left(\frac{\lambda_{2}}{\lambda_{3}}\right) \mu_{1}^{2} & \left(\Delta \lambda_{2}>0\right) .
\end{array}
$$

The first condition, Eq. 2.10), is a restatement of $\Delta \lambda_{1}<0$, which is necessary for the "standard" electroweak minimum $\left(v_{1}, 0\right)$ to be locally stable. The second condition, Eq. (2.11), ensures that this minimum is a global minimum at $T=0$, deeper than the exotic minimum at $\left(0, v_{2}\right)$. The third condition, Eq. (2.12), is equivalent to Eq. (2.9) and implies that the exotic $\varphi_{2}$ direction is likely to be destabilized before the $\varphi_{1}$ direction. The fourth condition, Eq. (2.13), corresponds to $\Delta \lambda_{2}>0$ and the absence of a barrier between $\left(v_{1}, 0\right)$ and $\left(0, v_{2}\right)$ at zero temperature.

In Fig. 2, we illustrate the region satisfying all these conditions. We fix $\lambda_{3}=0.1, y_{T}=0.2$ with $N=3$, and set $\lambda_{1}$ and $\mu_{1}^{2}$ such that $v_{1}=246 \mathrm{GeV}$ and $m_{r_{1}}=125 \mathrm{GeV}$ (where $r_{1}$ is the real scalar fluctuation around $\left.\left(v_{1}, 0\right)\right)$. The upper hatched region is excluded because it lacks a stable electroweak minimum. In the shaded regions, the conditions of Eqs. 2.10, 2.11, 2.12) are satisfied, with the requirement of Eq. (2.13) also realized in the darker shaded region. A bigger portal coupling $\lambda_{3}$ would lead to a larger tree-level barrier and therefore decrease the area of this region via Eq. 2.13). Increasing the value of $y_{T}$ or $N$ enhances thermal corrections in the $\phi_{1}$ direction, making it easier to satisfy $T_{c}^{1}<T_{c}^{2}$ (see Eq. (2.12)), which enlarges the wedge region. We will see below that when these considerations are applied to realistic theories of electroweak symmetry breaking, the parameter regions supporting a two-stage transition amenable to EWBG mostly correspond to the darker shaded region in this figure, where all four conditions are satisfied.

\section{Two Stages with Two Higgs Doublets}

Armed with the general considerations of the previous section, we turn next to realistic Higgs sectors, focusing primarily on a restricted inert two-Higgs-doublet model. We show that a strongly first-order electroweak-symmetry-breaking phase transition can occur first in the direction of the inert doublet, followed by an efficient second transition to the standard Higgs vacuum. We also discuss certain technical aspects of the calculation, and comment on a related extension with a new electroweak triplet scalar. 


\subsection{Methodology}

Most of our analysis will focus on the inert doublet model, consisting of the SM Higgs doublet $H$ together with an inert doublet $\Phi$ with no direct couplings to the SM fermions. The tree-level scalar potential is

$$
\begin{aligned}
V(H, \Phi)=- & m_{H}^{2} H^{\dagger} H-m_{\Phi}^{2} \Phi^{\dagger} \Phi+\frac{\lambda}{2}\left(H^{\dagger} H\right)^{2}+\frac{\lambda_{\Phi}}{2}\left(\Phi^{\dagger} \Phi\right)^{2} \\
& +\lambda_{\Phi H}\left(H^{\dagger} H\right)\left(\Phi^{\dagger} \Phi\right)+\tilde{\lambda}_{\Phi H}\left(\Phi^{\dagger} H\right)\left(H^{\dagger} \Phi\right) \\
& +\frac{\lambda_{5}}{2}\left[\left(\Phi^{\dagger} H\right)^{2}+\text { h.c. }\right] .
\end{aligned}
$$

This is the most general potential allowed when $\Phi$ is odd under a $\mathbb{Z}_{2}$ symmetry. This parity also forbids renormalizable couplings of $\Phi$ to SM fermions. A larger global $U(1)$ symmetry acting on $\Phi$ is present in the limit $\lambda_{5} \rightarrow 0$ [42. If both $\lambda_{5}=0=\tilde{\lambda}_{\Phi H}$, the global symmetry is enhanced further to an $S U(2)$ acting on $\Phi[42]]^{3}$

Motivated by the SM-like Higgs boson observed at the LHC [43, 44 and the consistency of the SM with precision electroweak tests, we will focus exclusively on parameters where only $H$ has a VEV today: $\langle H\rangle=v / \sqrt{2}=(246 / \sqrt{2}) \mathrm{GeV}$ and $\langle\Phi\rangle=0$. Negative quadratic mass parameters are assumed (corresponding to $m_{H}^{2}, m_{\Phi}^{2}>0$ ), as well as positive values of $\lambda, \lambda_{\Phi}$, and $\lambda_{\Phi H}$. For simplicity, we also take $\lambda_{5} \rightarrow 0$, and $\tilde{\lambda}_{\Phi H}<0$ with $\left|\tilde{\lambda}_{\Phi H}\right| \ll \lambda_{\Phi H}$. We will see that these choices are close to optimal for generating a two-stage electroweak phase transition, while a small but negative value of $\tilde{\lambda}_{\Phi H}$ also forces the charged components of the exotic doublet to be slightly heavier than the neutral ones.

The thermal evolution of the theory is tracked by computing the one-loop thermal effective potential $V_{\text {eff }}^{1}(h, \phi, T)$ in the Landau gauge $(\xi=0)$, where $h$ and $\phi$ are the canonicallynormalized background field values corresponding to the real neutral components of $H \rightarrow$ $h / \sqrt{2}$ and $\Phi \rightarrow \phi / \sqrt{2}$. We further improve the effective potential using the renormalization group with two-loop beta functions and anomalous dimensions when determining the couplings in terms of experimental measurements at scales $m_{Z}$ and $m_{t}$, with the relevant (one-loop) expressions for these collected in Appendix A. The top Yukawa coupling is determined from the pole mass including SM strong and weak threshold corrections of order $\alpha_{s}^{3}, \alpha_{w}$. The Higgs quartic and mass parameters are fixed by requiring the correct Higgs VEV and physical mass, which we take to be $v=246 \mathrm{GeV}$ and $m_{h}=125 \mathrm{GeV}$ [45]. The latter is matched to a zero of the neutral Higgs 1PI two-point function at $p^{2}=m_{h}^{2}$, the 1PI function being equal to the second derivative of the effective potential with respect to $h$ (yielding the zero momentum contribution) plus a finite momentum correction obtained from the general formulae of Ref. [46].

The negative quadratic coefficients of $H$ and $\Phi$ generate imaginary parts in the effective

\footnotetext{
${ }^{3}$ This global $S U(2)$ applies to the scalar potential, but is broken explicitly in the full Lagrangian by the gauging of $S U(2)_{L}$. Even so, the global $S U(2)$ can still be a useful approximate symmetry.
} 
potential at low temperatures and small field values. To minimize these effects, we resum the one-loop zero-temperature corrections for the Goldstone self-energies [47, 48] as well as the dominant finite temperature corrections for all bosonic self-energies [49, 50]. The thermal daisy resummation is known to improve convergence of the thermal corrections for small values of the effective bosonic masses; details of the temperature corrections to the bosonic masses are given in Appendix B.

Second-order phase transitions are straightforward to analyze using only the thermal effective potential, but first-order transitions require more care. Such transitions can occur when two local minima, $(h, \phi)=\left(v_{1}, w_{1}\right)$ and $\left(v_{2}, w_{2}\right)$, are present at the same temperature. In this case, the critical temperature $T_{c}$ is defined by the relation

$$
V_{\text {eff }}^{1}\left(v_{1}, w_{1} ; T_{c}\right)=V_{\text {eff }}^{1}\left(v_{2}, w_{2} ; T_{c}\right),
$$

where $V_{\text {eff }}^{1}$ includes the resummations described above. Suppose the system starts in the $\left(v_{2}, w_{2}\right)$ vacuum just above $T_{c}$, and the $\left(v_{1}, w_{1}\right)$ minimum becomes deeper than the first as $T$ falls below $T_{c}$. In this case, the system may transition to the second vacuum at some lower temperature $T_{n}$ by thermal fluctuations or quantum tunneling through the nucleation of bubbles of the lower-energy phase.

We analyze such tunneling transitions using the CosmoTransitions package [51], modified to ensure that the thermal corrections are evaluated appropriately for negative values of their arguments. When more than one minimum is present, the package is also used to compute the multifield tunneling rates and nucleation temperatures. The nucleation temperature of a first-order transition is the temperature below which the bubble nucleation rate overcomes the expansion rate of the Universe, allowing the transition to complete. When thermal tunneling dominates the formation of bubbles, this occurs when $S_{3} / T_{n} \lesssim 140[52$, where $S_{3}$ is the three-dimensional Euclidean action of the instanton corresponding to a thermal transition between the relevant vacua. In all cases, the relevant temperatures are large enough such that thermal transitions are more efficient than $O(4)$-symmetric quantum tunneling.

Let us also mention that two-stage electroweak phase transitions have been studied in the inert two-Higgs doublet model in Ref. [53] and more generally in Ref. [54]. It was also noted in Ref. [55] that non-inert vacua may appear due to large quantum corrections. The inert doublet model has also been applied to generate a strongly first-order Higgs-driven one-step electroweak phase transition suitable for EWBG in Refs. [55 59]. As far as we can tell, EWBG in a two-step transition has not been studied previously in this context.

\subsection{Parameter Scans}

Following the methods described above, we have analyzed the inert doublet model of Eq. (3.1) by scanning over the model parameters and searching for acceptable two-stage phase transitions. For each parameter point, we start at a very high temperature and track the evolution 

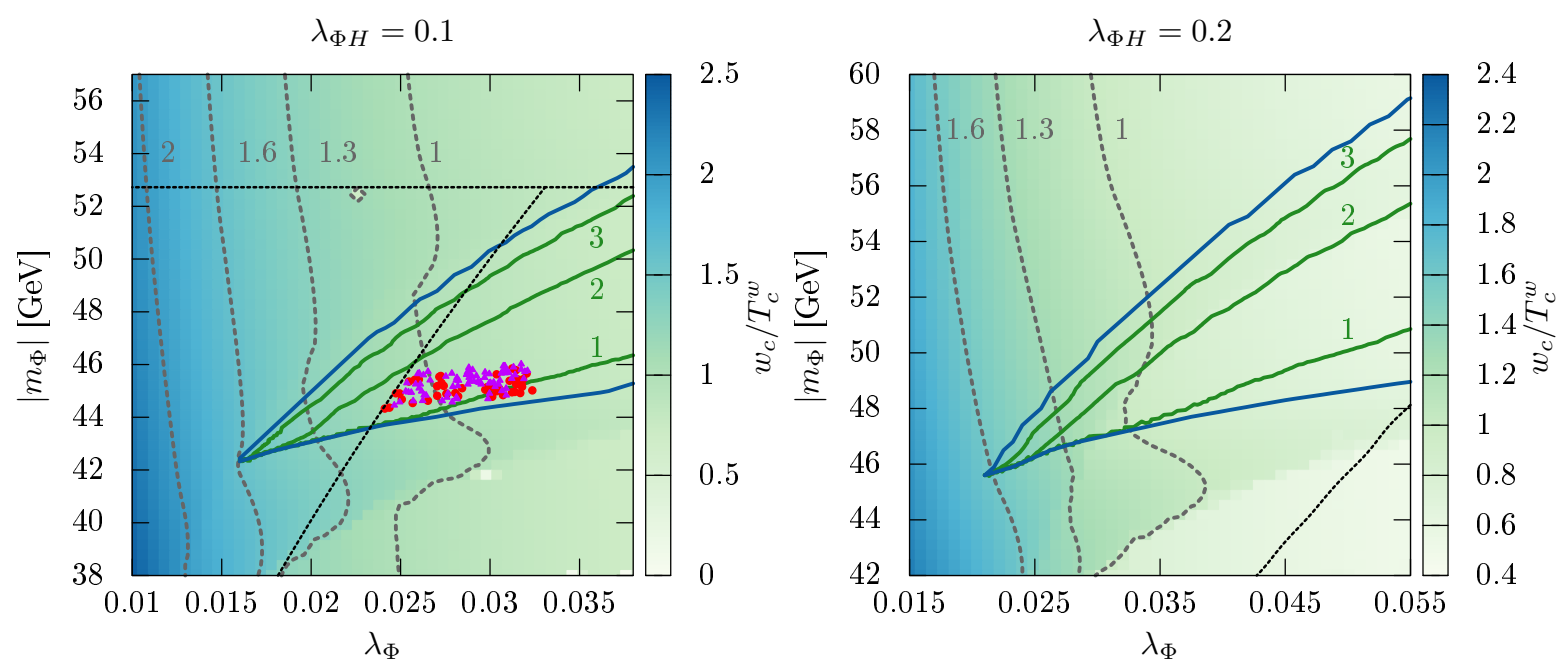

Figure 3: Parameter points leading to a two-stage electroweak phase transition amenable to EWBG in the $\lambda_{\Phi}-m_{\Phi}$ plane for $\lambda_{\Phi H}=0.1$ (left) and $\lambda_{\Phi H}=0.2$ (right). The purple triangles mark points where the first transition in the $\phi$ direction is strongly first-order and the second is second- or weakly first-order; the red dots correspond to both transitions being strongly first-order, with the second transition completing efficiently. The solid blue lines show the boundaries of the wedge region discussed in the text, while the colour shading and gray dashed contours show the estimated strength of the first transition based on the $w_{c} / T_{c}^{w}$ criterion. To the left of the diagonal black line, two minima exist at tree-level. Above the horizontal black line, the standard Higgs vacuum is unstable at $T=0$. This region is not visible in the parameter space shown for $\lambda_{\Phi H}=0.2$.

of the potential down to determine the order and structure of the cosmological phase transitions. The free parameters in our scans are $m_{\Phi}^{2}, \lambda_{\Phi}, \lambda_{\Phi H}$, and $\tilde{\lambda}_{\Phi H}$, with the other parameters fixed in terms of $m_{h}$ and $v$ to guarantee a (local) minimum at $T=0$ with $\phi=w=0$, $h=v=246 \mathrm{GeV}$, and $m_{h}=125 \mathrm{GeV}$. We focus on $\lambda_{\Phi} \in[0.01,0.055], \sqrt{m_{\Phi}^{2}} \in[38,60] \mathrm{GeV}$, for fixed values of $\lambda_{\Phi H} \in\{0.1,0.2\}$ and $\tilde{\lambda}_{\Phi H}=-0.001$.

In Fig. 3 we show the main results of our parameter scans in the $\lambda_{\Phi}-m_{\Phi}$ plane for $\lambda_{\Phi H}=0.1$ (left) and $\lambda_{\Phi H}=0.2$ (right). The solid red and purple dots correspond to specific parameter points that give rise to a two-stage electroweak phase transition that is potentially viable for EWBG. For these points, the first transition occurs in the $\phi$ direction and is strongly first-order with

$$
w_{n} / T_{n}^{w} \geq 1
$$

where $T_{n}^{w}$ is the nucleation temperature computed with CosmoTransitions, and $w_{n}$ is the VEV of $\phi$ in the symmetry-breaking vacuum when this occurs. Among the parameter points where the first transition is strongly first-order, we also demand that the second transition completes efficiently. As discussed above, first-order transitions complete when the bubble 
nucleation rate overcomes the expansion rate of the Universe, which for thermal tunneling processes roughly takes place when $S_{3} / T_{n}<140$. The other features of Fig. 3 will be explained below.

The solid blue and dashed black lines in Fig. 3 correspond to the conditions of Eqs. 2.102.13) derived for the toy model when applied to this theory. To translate these conditions to the inert doublet model, we note that the tree-level potential of Eq. (3.1) projected onto the neutral real scalar components of $H$ and $\Phi$ coincides with the (tree-level) toy model presented in Section 2.3. The specific parameter identifications are

$$
\begin{aligned}
& m_{H}^{2} \rightarrow \mu_{1}^{2}, \quad m_{\Phi}^{2} \rightarrow \mu_{2}^{2}, \\
& \lambda \quad \rightarrow \lambda_{1}, \quad \lambda_{\Phi} \rightarrow \lambda_{2}, \quad\left(\lambda_{\Phi H}+\tilde{\lambda}_{\Phi H}\right) \rightarrow \lambda_{3},
\end{aligned}
$$

and the $a$ parameters of Eq. 2.8 now include gauge boson contributions. As in Fig. 2, the top of the wedge region enclosed by the thick solid blue lines bounds the region where the standard Higgs minimum is deeper at $T=0$. Above the lower boundary of the wedge the $\phi$ direction is destabilized before the $h$ direction to leading order in the thermal expansion of the potential. The upper horizontal black line in the left panel $\left(\lambda_{\Phi H}=0.1\right)$ shows where the standard vacuum disappears entirely, while to the right of the black diagonal line the standard Higgs vacuum is the only one present at $T=0$. The horizontal line is also present for $\lambda_{\Phi H}=0.2$, but it is not visible within the parameter ranges shown.

The shading and gray dashed contours in Fig. 3 correspond to $w_{c} / T_{c}^{w}$, defined to be the ratio of the $\phi \mathrm{VEV}$ at the local extremum in the $(0, \phi)$ direction to the critical temperature $T_{c}^{w}$ (at which $(0, \phi)$ becomes degenerate with the origin). Note that this definition does not require that the extrema at $\left(0, w_{c}\right)$ or the origin be local minima, and thus the ratio $w_{c} / T_{c}^{w}$ does not necessarily correspond to a physical phase transition as it is usually applied. However, such an interpretation can be made within the wedge region bounded by the solid blue lines discussed above. Within the wedge, we also show contours of $v_{c} / T_{c}^{v}$ as solid green lines, defined similarly to be the ratio of the $h \mathrm{VEV}$ at the local extremum $\left(v, w^{\prime}\right)$ at the critical temperature $T_{c}^{v}$ (when it becomes degenerate with the local minimum at $(0, w)$ ).

The EWBG-viable points in Fig. 3 are also colour coded according to whether the second phase transition is strongly first-order (red), or second-order or weakly first-order (purple). For the red points, we have checked that the second transition completes efficiently using CosmoTransitions. The second transition in this case will inject some entropy into the cosmological plasma and dilute the the baryon asymmetry produced at the first transition. We have verified that this entropy injection is small for all of the points considered, typically resulting in a percent-level dilution of the asymmetry. For the purple points, we have checked that the combination $\sqrt{h^{2}+\phi^{2}} / T$ remains greater than unity over the course of the transition to ensure a sufficient suppression of sphaleron washout during the second transition [41]. An example of this situation is shown in Fig. 4. The left panel of the figure shows that two minima exist away from the origin at high temperature $T=100 \mathrm{GeV}$. In the right 

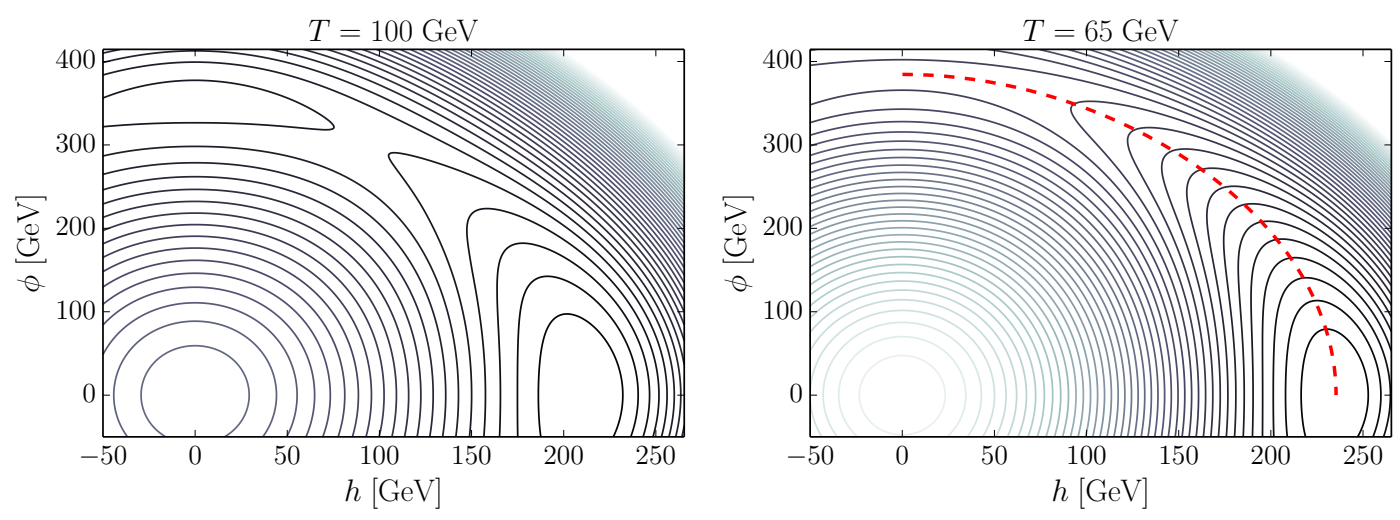

Figure 4: Contour plot of the one-loop effective potential in the $\phi-h$ plane of the inert doublet model for $\lambda_{\Phi H}=0.1, \tilde{\lambda}_{\Phi H}=-0.001, \lambda_{\Phi}=0.0285, m_{\Phi}^{2}=(45.3 \mathrm{GeV})^{2}$, at $T=100$ $\mathrm{GeV}$ (left) and $T=65 \mathrm{GeV}$ (right). Two local minima are present at $T=100 \mathrm{GeV}$ while only the standard electroweak minimum is stable at $T=65 \mathrm{GeV}$. The dashed red line shows the trajectory of the second order transition from the exotic to the SM Higgs vacuum.

panel, we see that at a lower temperature of $T=65 \mathrm{GeV}$, the $\phi \neq 0$ minimum becomes a saddle point and the field rolls down to the $h \neq 0$ vacuum following the field trajectory shown by the dashed red line.

Note that the solid red and purple dots in Fig. 3 featuring a two-stage electroweak phase transition suitable for EWBG are found only in the left $\lambda_{\Phi H}=0.1$ panel, and all of them lie within the wedge region and to the right of the diagonal dashed boundary line where $\Delta \lambda_{2}>0$. These features exemplify the general expectations derived from the toy model of Section 2.3. To the left of the diagonal boundary, a tree-level barrier between the local minima along the $\phi$ and $h$ directions persists at $T=0$, and the second transition from the exotic to the standard electroweak vacuum never completes. The viable points also lie in the leftmost part of this subset of the wedge region where $w_{c} / T_{c}^{w} \gtrsim 1$ since this ratio is often a good estimate for $w_{n} / T_{n}^{w}$. These two considerations also explain why no points are found for $\lambda_{\Phi H}=0.2$ (right panel of Fig. 3); the diagonal boundary for $\lambda_{2}>0$ now lies further off to the right where $w_{c} / T_{c}^{w}$ is very small, and it is not possible for the first transition to be strongly first-order, while also having the second transition complete efficiently.

More generally, we only find two-stage electroweak phase transitions suitable for EWBG in a limited range $\lambda_{\Phi H} \sim 0$.1. Significantly larger values face the same challenge as $\lambda_{\Phi H}=0.2$, with the second transition not completing efficiently. Smaller values of $\lambda_{\Phi H} \lesssim 0.07$ are also problematic because they destabilize the standard Higgs minimum in the parameter region where the exotic direction is destabilized first (corresponding to lowering the horizontal black line in Fig. 3 related to the condition of Eq. (2.10) below the lower boundary of the wedge). We expect that a wider range of $\lambda_{\Phi H}$ is possible with additional matter coupling to $H$ or $\Phi$. 


\subsection{Comments on Gauge Dependence}

The viable points in Fig. 3 rely primarily on the non-analytic gauge boson contributions to the thermal effective potential to drive the first transition in the $\phi$ direction to be first-order. The challenge presented by these contributions is that they are gauge-dependent [5, 6], and they lead to a gauge dependence in the ratio $w_{n} / T_{n}^{w}$ used to estimate the strength of the first phase transition. For this reason, some care must be taken in interpreting the results of the calculation, which were obtained in the Landau gauge $(\xi=0)$.

To check the robustness of our findings, we have also analyzed the transition strength of several scan points following the gauge-invariant prescription of Ref. [5] to first-order in the loop expansion. While the critical temperatures change substantially (reflecting the breakdown of the loop expansion near $T_{c}$ ), we find that viable two-step transitions still typically occur for the points shown, although usually with a reduced strength. Given the significant uncertainty in determining the rate of non-perturbative baryon washout in the broken phase [5], these points remain very plausible candidates for two-stage EWBG.

Let us also mention that the effective potential for $\phi$ along the $h=0$ axis closely mirrors the potential for the SM Higgs in the limit $m_{h} \ll m_{W}$, which can be similarly driven strongly first-order by gauge boson loop effects. This scenario has been studied in great detail, with the literature including a number of non-perturbative studies [7,8] which do not suffer from gauge-dependence. The strength of the $\phi$ transition we find matches reasonably well with those reported in these previous investigations, giving us further confidence in the reliability of our results.

\subsection{Comments on a Triplet Extension}

To conclude this section, we review the results for the real triplet extension of the Standard Model studied in Ref. [31] and we compare them to the inert doublet model discussed above. The triplet does not couple to the SM fermions, thereby allowing the Higgs to receive stronger thermal corrections than the exotic states, in line with the general requirements for viable models with two-stage transitions delineated in Section 2.2. However, unlike the inert doublet case, the triplet also couples more strongly than the Higgs to the electroweak gauge bosons and thus has a relatively enhanced contribution to its thermal mass from these states. Hence, we expect a more limited parameter space for which two strongly first-order transitions are feasible relative to the models we have considered thus far.

The model considered in Ref. [31] consists of the SM plus a real $S U(2)_{L}$ triplet, $\Sigma=$ $\left(\Sigma^{1}, \Sigma^{2}, \Sigma^{3}\right)$, with zero hypercharge $Y=0$ and the relevant interactions given by

$$
V(H, \Sigma)=-m_{H}^{2} H^{\dagger} H-\frac{1}{2} m_{\Sigma}^{2} \Sigma \cdot \Sigma+\frac{\lambda}{2}\left(H^{\dagger} H\right)^{2}+\frac{\lambda_{\Sigma}}{4}(\Sigma \cdot \Sigma)^{2}+\frac{\lambda_{\Sigma H}}{2}\left(H^{\dagger} H\right)(\Sigma \cdot \Sigma) .
$$




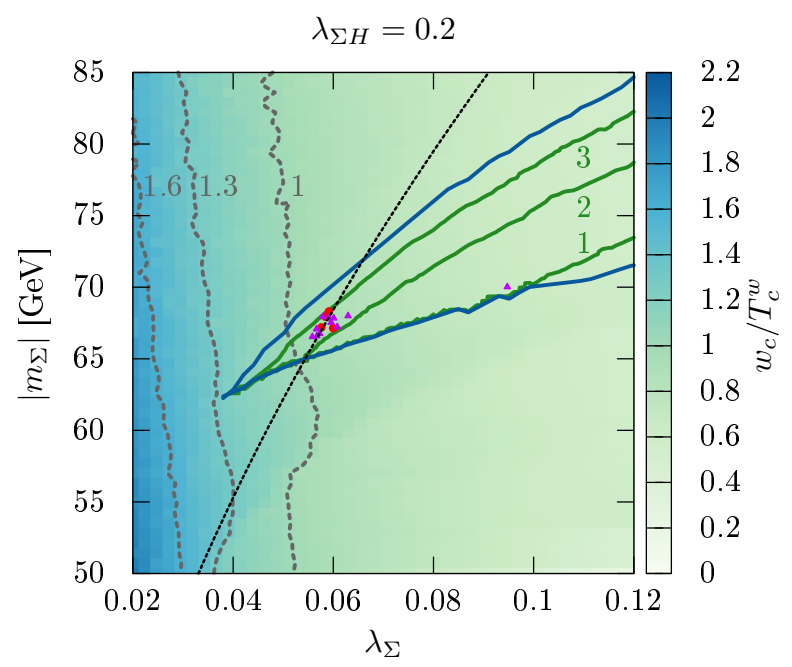

Figure 5: Parameter points leading to a two-stage electroweak phase transition amenable to EWBG in the $\lambda_{\Sigma}-m_{\Sigma}$ plane for the real triplet extension of the SM with $\lambda_{\Sigma H}=0.2$. The purple triangles mark points where the first transition in the $\phi$ direction is strongly first-order and the second is second- or weakly first-order; the red dots correspond to both transitions being strongly first-order, with the second transition completing efficiently. The solid blue lines show the boundaries of the wedge region discussed in Section 3.2, while the colour shading and gray dashed contours show the estimated strength of the first transition based on the $w_{c} / T_{c}^{w}$ criterion. Points to the left of the diagonal line exhibit two minima separated by a barrier at $T=0$.

Projecting these fields onto their real neutral components, this potential can be matched to the toy model of Section 2.3 with the identifications

$$
\begin{array}{llll}
m_{H}^{2} & \rightarrow \mu_{1}^{2}, & m_{\Sigma}^{2} & \rightarrow \mu_{2}^{2}, \\
\lambda & \rightarrow \lambda_{1}, & \lambda_{\Sigma} & \rightarrow \lambda_{2} / 2, \quad\left(\lambda_{\Phi H}+\tilde{\lambda}_{\Phi H}\right) \rightarrow \lambda_{3} .
\end{array}
$$

The thermal mass corrections must also be modified appropriately and can be easily deduced from Ref. 31.

We have investigated this theory using the same techniques as for the inert doublet model by scanning over the parameters $\lambda_{\Sigma}$ and $m_{\Sigma}^{2}$ with fixed $\lambda_{\Sigma H} \in\{0.1,0.3\}$. Figure 5 shows the results of this scan for $\lambda_{\Sigma H}=0.2$, with the plot format directly analogous to Fig. 3 . As before, the purple triangles and red dots correspond to parameter points that are suitable for EWBG where the first phase transition in the exotic $\Sigma$ direction is strongly first-order, with the second transition being strongly first-order (purple triangles), or second-order or weakly first-order (red dots).

These results show many similarities to the findings for the inert doublet model and are consistent with the toy model expectations. As before, the potentially viable points all lie 
within the wedge region bounded by the solid blue lines. They also lie mostly to the right of the dotted black diagonal line where no exotic minimum is present at zero temperature. In general, we also find fewer points in the triplet case that are suitable for two-stage EWBG, as well as larger allowed values for $\lambda_{\Sigma H}$ and $m_{\Sigma}^{2}$. This can be understood in terms of the stronger stabilization of the triplet direction from thermal gauge boson loops. To destabilize the triplet direction before the Higgs, a larger $m_{\Sigma}^{2}$ is needed, which requires in turn a larger $\lambda_{\Sigma H}$ to drive the transition first-order.

The results of our scan also appear to be consistent with the findings of Ref. [31], although we also focus on a slightly different region of the parameter space. Relative to that work, we impose a stronger requirement on the strength of the first phase transition in the $\Sigma$ direction, although the weaker transitions considered in Ref. [31] are potentially also consistent with EWBG given the significant uncertainties associated with determining the extent of baryon washout by non-perturbative transitions [5]. After the first transition in this model, baryon washout can occur both through sphalerons, and the scattering of baryons with the $S U(2)_{L}$ monopoles that can arise in the broken-triplet phase [31]. The approximate condition for the suppression of sphalerons is similar to the usual electroweak doublet, with $w_{n} / T_{n} \gtrsim 0.86$ at leading order [41]. The corresponding requirement for monopole suppression is more uncertain, but it is argued in Ref. [31] to be similar to that for sphaleron suppression. With our stronger condition of $w_{n} / T_{n}^{w} \geq 1$, the coupling value $\lambda_{\Sigma H}=0.2$ is about as large as possible.

\section{Phenomenological Constraints and the Need for More}

In the previous section, we showed that two-stage phase transitions suitable for EWBG can occur in models with an additional inert Higgs doublet. We turn next to investigate whether the model parameter space of these scenarios can be consistent with existing experimental constraints. In general, we find that all the parameter points that exhibit two-stage phase transitions are in conflict with observation due to the existence of light inert states in the spectrum. To correct this, we construct a mild singlet extension of the inert doublet model that drives up the inert doublet mass without significantly modifying the two-stage electroweak phase transition.

\subsection{Mass Spectrum of the Inert Model}

The inert doublet model presented above has four new physical degrees of freedom, in addition to the usual SM states. They consist of neutral and charged complex scalars, $\phi^{0}$ and $\phi^{ \pm}$. At tree-level, their masses are

$$
m_{\phi^{0}}^{2}=\frac{1}{2}\left(\lambda_{\Phi H}+\widetilde{\lambda}_{\Phi H}\right) v^{2}-m_{\Phi}^{2}
$$




$$
m_{\phi^{ \pm}}^{2}=\frac{1}{2} \lambda_{\Phi H} v^{2}-m_{\Phi}^{2}
$$

where $v=246 \mathrm{GeV}$. The small and slightly negative $\tilde{\lambda}_{\Phi H}$ used in the analysis above implies that the charged states are slightly heavier than the neutral ones. Note as well that a non-zero $\lambda_{5}$ coupling in Eq. (3.1) would split the complex $\phi^{0}$ state into a scalar $S$ and a pseudoscalar $A$ separated in mass by $\Delta m^{2}=\lambda_{5} v^{2}$. We will focus on the $\lambda_{5} \rightarrow 0$ limit, but we will comment on those cases where a small non-zero value can significantly modify the phenomenology.

Comparing these expressions to the parameter values found to yield two-stage transitions, all the points that are consistent with EWBG lead to new scalars that are very light, $m_{\phi^{0, \pm}} \lesssim 35 \mathrm{GeV}$. We expect this to hold beyond the specific parameter ranges studied above. Larger scalar masses would require larger values of $\lambda_{\Phi H}$ and smaller values of $m_{\Phi}^{2}$. However, increasing $\lambda_{\Phi H}$ makes it more difficult for the second transition to complete (corresponding to Eq. (2.13) with $\lambda_{3} \rightarrow \lambda_{\Phi H}$ ), while decreasing $m_{\Phi}^{2}$ tends to destabilize the standard Higgs $h$ direction before the exotic $\phi$ direction (corresponding to Eq. (2.12) with $\mu_{2}^{2} \rightarrow m_{\Phi}^{2}$ ).

In the analysis to follow, we will see that inert scalar masses below about $63 \mathrm{GeV}$ are inconsistent with current experimental bounds. With this in mind, we will study the limits on the theory while allowing for an additional contribution to the scalar masses, which we will parametrize according to

$$
m_{\phi^{0, \pm}}^{2} \rightarrow m_{\phi^{0, \pm}}^{2}+\Delta^{2}
$$

We will return later to exhibit a singlet extension of the inert doublet model that provides such an enhancement without significantly changing the phase transition structure or the other direct constraints on the theory.

\subsection{Precision Electroweak Tests}

The states from the new electroweak doublet can modify precision electroweak observables through their oblique loop corrections to the weak vector bosons [60,61]. Explicit expressions for these effects in the inert doublet model can be found in Ref. [62], and the most important constraint usually comes from $\Delta T$. Evaluating them, we find only very mild shifts in the oblique parameters that are consistent with current limits even for $\Delta^{2}=0$. This can be

understood from the custodial $S U(2)$ of the theory in the limit of $\tilde{\lambda}_{\Phi H} \rightarrow 0$ [42], which is realized to a good approximation for the small value $\tilde{\lambda}_{\Phi H}=-0.001$ chosen in our analysis.

The new scalar states can alter the behaviour of the $W$ and $Z$ vector bosons in a more direct way by opening up new decay channels. To be consistent with data, new vector boson decay channels must be nearly or completely kinematically forbidden, implying

$$
2 m_{\phi^{0}}, 2 m_{\phi^{ \pm}} \gtrsim m_{Z}, \quad m_{\phi^{0}}+m_{\phi^{ \pm}} \gtrsim m_{W}
$$


The first limit comes from the invisible decay width of the $Z$, or from searches for $Z \rightarrow$ $f \bar{f}+\nu \bar{\nu} .63,64$. The second limit above follows from the precisely measured decay width of the $W[65]$.

\subsection{Direct Collider Searches}

Searches for supersymmetry at LEP can be applied to derive limits on the new scalars. A reinterpretation of the OPAL search for charginos 66, 67] suggests $m_{H^{ \pm}} \gtrsim 70 \mathrm{GeV}$ [68, although this limit disappears for mass splittings between the charged and neutral states below about $5 \mathrm{GeV}$. Similarly, the LEP search for neutralinos $\left(\chi_{1}^{0} \chi_{2}^{0}\right)$ can also be recast into a limit on the production cross-sections for the neutral inert scalar $\phi^{0}$. If the mass splitting between the scalar and pseudoscalar components of $\phi^{0}$ is at least $\Delta m>8 \mathrm{GeV}$, the bound is $m_{\phi^{0}} \gtrsim 100 \mathrm{GeV}[69]$. However, for the small mass splittings $\Delta m<8 \mathrm{GeV}$ that arise in the $\lambda_{5} \rightarrow 0$ limit considered here, this bound disappears and the only relevant LEP limit on the neutral state is $m_{\phi^{0}} \gtrsim m_{Z} / 2$.

Data from the LHC has also been applied to constrain new inert doublets. These analyses typically use many of the same methods as for chargino and neutralino searches $63,64,70,73$. New bounds have been obtained beyond LEP, but they do not provide a useful constraint on the model parameter space in the nearly-degenerate limit considered here [70 74$]$.

\subsection{Higgs Boson Decays}

The presence of new electroweakly-charged scalars can have a significant effect on how the SM-like Higgs boson decays. These effects can be direct if the Higgs decays into pairs of the new states, or they can be indirect from the new scalars running in loops. We examine both possibilities here.

Direct decays of the Higgs into the scalars can occur if they are light enough. The decay width to $\phi^{0}$ is 68

$$
\Gamma\left(h \rightarrow \phi^{0} \phi^{0}\right)=\frac{\left(\lambda_{\Phi H}+\tilde{\lambda}_{\Phi H}\right)^{2}}{16 \pi} \frac{v^{2}}{m_{h}} \sqrt{1-\left(\frac{2 m_{\phi}}{m_{h}}\right)^{2}}
$$

Away from the kinematic threshold $m_{h}=2 m_{\phi^{0}}$, this mode (and possibly its charged counterpart) completely dominates the Higgs branching for $\left(\lambda_{\Phi H}+\tilde{\lambda}_{\Phi H}\right) \gtrsim 0.05[74$, and is constrained by current Higgs data to lie below $\left(\lambda_{\Phi H}+\tilde{\lambda}_{\Phi H}\right) \lesssim 7 \times 10^{-3}|75|$. Larger values of this coupling appear to be needed for a viable two-stage electroweak phase transition, so we will focus on masses above $m_{\phi^{0}} \gtrsim 63 \mathrm{GeV}$ where such decays are kinematically forbidden (on-shell). 

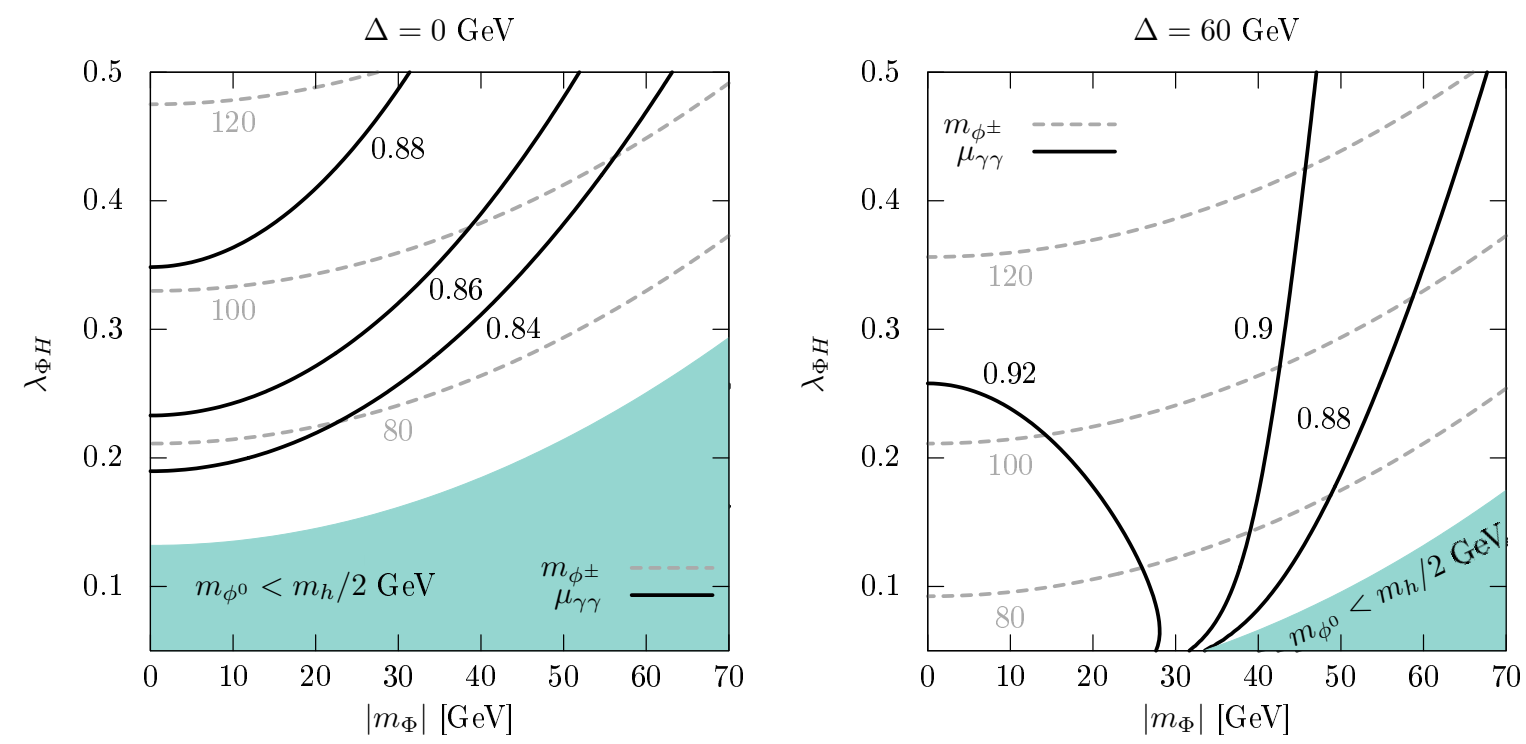

Figure 6: Contours showing deviations in the Higgs to diphoton signal rate $\mu_{\gamma \gamma}$ (solid black lines) and the charged scalar mass $m_{\phi^{ \pm}}$(dashed gray lines) in the $m_{\Phi}-\lambda_{\Phi H}$ plane of the inert doublet model with the mass correction parameter equal to $\Delta=0$ (left) and $\Delta=60 \mathrm{GeV}$ (right).

If the direct decays are closed, the most important effect of the new doublet on the SMlike Higgs is to modify its decay width to $\gamma \gamma$ and $\gamma Z\left[7678\right.$. In particular, the $\phi^{ \pm}$states will contribute a new loop to the amplitudes for these processes at a level that is similar to the dominant $W$ loop in the SM. The other Higgs decay modes relevant at the LHC will not be changed at leading order. Modifications to the branching ratios of the Higgs were computed using the formulae of Refs. 79 82], while the SM width was evaluated using HDECAY [83]. The results for the diphoton channel are shown in Fig. 6, where we show the deviation in the signal rate $\mu_{\gamma \gamma}=(\sigma \mathrm{BR}) /(\sigma \mathrm{BR})_{\mathrm{SM}}$ in the $\lambda_{\Phi}-m_{\Phi}$ plane for $\Delta=0$ (left) and $\Delta=60 \mathrm{GeV}$ (right). In both panels the deviations are generally consistent with the signal strengths observed by at ATLAS [84] and CMS [85],

$$
\mu_{\gamma \gamma}=1.17 \pm 0.27 \text { (ATLAS) }, \quad \mu_{\gamma \gamma}=1.14_{-0.23}^{+0.26}(\mathrm{CMS}) .
$$

Similar conclusions hold for the triplet case [31].

\subsection{Dark Matter}

The inert doublet model we have considered has an unbroken global $U(1)$ in the standard electroweak vacuum (in the $\lambda_{5} \rightarrow 0$ limit), and the lightest exotic state will therefore be stable and contribute to the density of dark matter. For $\tilde{\lambda}_{\Phi H}<0$, this state will be the neutral scalar $\phi^{0}$. The relic abundance of the lightest scalar in inert doublet models has 
been studied extensively in, e.g., Refs. [55, 64, 74, 86, 88], and we restrict ourselves to a few brief comments.

For $\left|\tilde{\lambda}_{\Phi H}\right| \lesssim m_{\phi^{0}}^{2} / v^{2}$, the charged and neutral states are close in mass and produce a very small thermal relic density through coannihilation [68]. As the neutral-charged mass splitting increases (with a heavier charged state), the relic density increases as well but still remains well below the full dark matter density thanks to efficient annihilation through the $Z^{0}$ to fermions [62]. The relic density of the lightest state will increase further if the global $U(1)$ symmetry is broken to $\mathbb{Z}_{2}$ by introducing a small $\lambda_{5}$ coupling; this separates the scalar and pseudoscalar components of $\phi^{0}$ and moderates the annihilation via the $Z^{0}[62$. Since the required splittings are small, we expect that all these possibilities can be realized while maintaining a two-step electroweak phase transition consistent with EWBG.

The lightest inert scalar is also strongly constrained by direct searches for dark matter, even if the scalar relic density is well below the full dark matter density. This is especially true when the scalar and pseudoscalar components of $\phi^{0}$ are degenerate $\left(\lambda_{5} \rightarrow 0\right)$, since there is an extremely strong $\phi^{0}$-nucleon interaction mediated by $Z^{0}$ exchange. When $\lambda_{5}$ is turned on, the $Z$ couples exclusively to the scalar-pseudoscalar combination and the scalarnucleon scattering is inelastic [89]. Thus, it becomes irrelevant to direct detection searches for mass splittings above a few hundred $\mathrm{keV}$. The next most important contribution to nucleon scattering comes from Higgs exchange [62], and even this is too large compared to current limits from XENON100 [90] and LUX [91] for $\left(\lambda_{\Phi H}+\lambda_{\Phi H}+\lambda_{5}\right) / 2 \gtrsim 0.02$ and $m \in[10,100] \mathrm{GeV}$ assuming a local scalar relic density of $\rho_{\phi}=0.3 \mathrm{GeV} / \mathrm{cm}^{3}$ [4].

Taken together, these results suggest that it is unlikely for the lightest scalar to make up all of the dark matter while satisfying the limits from direct detection and producing a viable two-stage electroweak transition. On the other hand, the lightest scalar could make up a small fraction of the dark matter density provided it is neutral with at least a small mass splitting ( $\gtrsim 100 \mathrm{keV}$ ) between its scalar and pseudoscalar components. Alternatively, the lightest scalar could be destabilized by introducing an explicit soft breaking of the $\mathbb{Z}_{2}$ symmetry [37, 38], allowing the would-be dark matter to decay. If this breaking is small enough, the phase transition history is expected to be mostly unchanged.

\subsection{A Singlet Extension}

As discussed above, the exotic scalars $\phi^{0, \pm}$ are very light in the parameter regions found to yield a two-stage electroweak phase transition suitable for EWBG. Such scalars are excluded by experimental measurements of weak vector boson and Higgs decays. We parametrized an additional contribution to the scalar masses by a factor $\Delta$ such that $m_{\phi^{0, \pm}}^{2} \rightarrow m_{\phi^{0, \pm}}^{2}+\Delta^{2}$. In this section we exhibit a simple singlet extension of the theory to generate such a $\Delta$ contribution that does not significantly disturb the electroweak phase transitions.

The potential in the extended theory for the scalar electroweak doublets and the new 


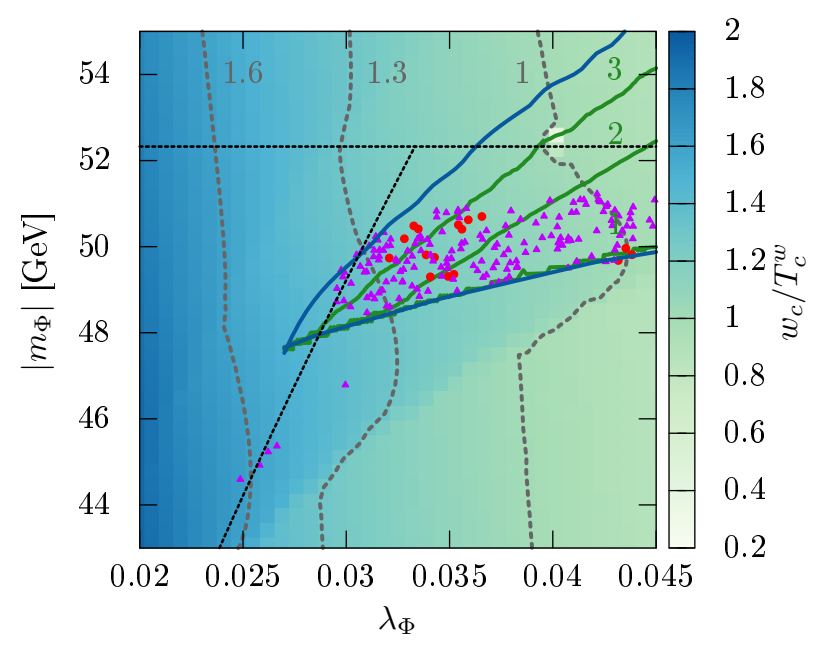

Figure 7: Parameter points leading to a two-stage electroweak phase transition amenable to EWBG in the $\lambda_{\Phi}-m_{\Phi}$ plane for the inert doublet model extended by a singlet $\lambda_{\Phi H}=0.1$. The remaining parameters are chosen as described in the text. The singlet field $S$ develops a nonzero $\mathrm{VEV}$ at $T_{c} \sim 20 \mathrm{GeV}$, well below the temperature of the transitions in the figure. The various contours and solid points are the same as in Figs. 3 and 5 .

real singlet $S$ is taken to be

$$
\begin{aligned}
V(H, \Phi, S)= & -m_{H}^{2} H^{\dagger} H-m_{\Phi}^{2} \Phi^{\dagger} \Phi+\frac{\lambda_{H}}{2}\left(H^{\dagger} H\right)^{2}+\frac{\lambda_{\Phi}}{2}\left(\Phi^{\dagger} \Phi\right)^{2} \\
& +\lambda_{\Phi H}\left(H^{\dagger} H\right)\left(\Phi^{\dagger} \Phi\right)+\tilde{\lambda}_{\Phi H}\left(\Phi^{\dagger} H\right)\left(H^{\dagger} \Phi\right)+\left[\frac{1}{2} \lambda_{5}\left(H^{\dagger} \Phi\right)^{2}+\text { h.c. }\right] \\
& -\frac{1}{2} m_{S}^{2} S^{2}+\frac{\lambda_{S}}{4 !} S^{4}+\frac{\lambda_{S H}}{2} H^{\dagger} H S^{2}+\frac{\lambda_{S \Phi}}{2} \Phi^{\dagger} \Phi S^{2},
\end{aligned}
$$

where possible additional terms can be forbidden with suitable symmetries. As before, we will invoke an approximate $U(1)$ symmetry to set $\lambda_{5} \rightarrow 0$. We will also set $\lambda_{S H} \rightarrow 0$ for now to avoid mixing with the SM Higgs, but we will return to non-zero values below. If the singlet develops a VEV,$S=v_{S}+\rho$, the masses of both the neutral and charged doublets $\phi^{0, \pm}$ will be modified by $m_{\phi^{0, \pm}}^{2} \rightarrow\left(m_{\phi^{0, \pm}}^{2}+\Delta^{2}\right)$ with

$$
\Delta^{2}=\frac{\lambda_{S \Phi}}{2} v_{S}^{2}=\frac{3 \lambda_{S \Phi}}{\lambda_{S}} m_{S}^{2}
$$

The mass of the real singlet excitation in the present vacuum is

$$
m_{\rho}^{2}=\frac{\lambda_{S}}{3} v_{S}^{2}=2 m_{S}^{2}
$$

In Fig. 7 we show parameter points in this extended theory that realize a two-step electroweak phase transition that is amenable to EWBG for the model parameters $\lambda_{\Phi H}=0.1$, 
$\lambda_{S}=0.001, m_{S}=4.63 \mathrm{GeV}, \lambda_{S \Phi}=0.72$, and $\lambda_{S H}=0$. For these points, the $S$ field is found to develop a VEV at temperatures near $T_{c}^{S} \sim 20 \mathrm{GeV}$, well below the temperatures of the other transitions. Thus, the singlet mostly decouples from the dynamics of the electroweak transitions for our choice of parameters, and its primary physical effect is to generate the mass contribution $\Delta^{2}$ at late times.

The distribution of EWBG-viable points in Fig. 7 is mostly similar to what was found for the pure inert doublet (Fig. 3) and triplet (Fig. 5), with most of the points in the wedge region to the right of the dashed black diagonal line. However, there is also a small population of viable dots in the lower left of Fig. 7 outside the wedge region. These points lie below the bottom of the wedge where the $h$ direction is expected to be destabilized before the $\phi$ direction based on the simple analysis of Section 2.3. The more complete analysis of the thermal evolution of the potential undertaken here shows that some points can avoid this criterion to an extent. Note as well that this second population, as well as a subset of points within the wedge, lie very close to, but to the left of the boundary where $\Delta \lambda_{2}=0$. For these points, a local minimum persists in the $\phi$ direction at zero temperature, but the barrier separating it from the standard electroweak vacuum may be small enough for tunneling to occur at an acceptable rate. The corresponding transitions would be weakly first-order.

While we have set $\lambda_{S H}=0$ in the analysis above, this limit is unnatural since loops will generate

$$
\Delta \lambda_{S H}(\mu) \sim \frac{\lambda_{S \Phi} \lambda_{\Phi H}}{(4 \pi)^{2}} \ln \left(\frac{\mu}{\mu_{0}}\right),
$$

where $\mu$ is the running scale and $\mu_{0}$ is a reference scale where $\lambda_{S H}$ vanishes. Similarly, the singlet self-coupling receives contributions from quantum corrections on the order of $\Delta \lambda_{S} \sim \lambda_{S \Phi}^{2} \ln \left(\mu / \mu_{0}\right) /(4 \pi)^{2}$, so naturalness sets an approximate lower bound on $\lambda_{S}$ as well. The phase transition picture described above will be preserved as long as $\lambda_{S}$ and $\lambda_{S H}$ are small, near the lower range consistent with naturalness. However, one could also imagine larger values where the singlet plays a key role in the other phase transitions.

A non-zero value of $\lambda_{S H}$ also gives rise to mixing between the singlet and the SM-like Higgs boson. This Higgs portal coupling allows the Higgs to decay to pairs of light singlets, and it opens a new decay channel for the singlet states [92,93]. For $m_{\rho}<m_{\phi}$, the dominant decay of the mostly-singlet state proceeds through the Higgs portal and will mirror the branching fraction a SM-like Higgs would have if $m_{h}=m_{\rho}$. Searches for rare $B$ decays imply very strong limits on $\lambda_{S H}$ for $m_{\rho}<m_{B}-m_{K}$ that typically rule out natural values of this coupling 94 96. For higher masses, the limits become much weaker, coming from a combination of LEP searches for a light Higgs boson (typically above the $b \bar{b}$ threshold) 95,96 , rare $\Upsilon$ decays [95], and low-mass dimuon searches at the LHC [95]. These typically allow for small but natural values of $\lambda_{S H}$.

Finally, let us note that this theory has a global $\mathbb{Z}_{2}$ symmetry under which $S \rightarrow-S$ that is spontaneously broken when $S$ gets a VEV. As it stands, this will lead to the formation of 
cosmologically problematic domain walls [97]. These can be eliminated without significantly modifying the electroweak phase transition structure by adding a very small explicit soft breaking of the $\mathbb{Z}_{2}$, or by promoting $S$ to a complex scalar charged under a hidden $U(1)_{x}$ gauge group.

\section{Conclusions}

Electroweak baryogenesis provides a well-motivated explanation of the observed baryon asymmetry that is in principle testable at present day experiments. As such, it is important to understand its possible realizations and the extent to which they can be probed. Indeed, many existing proposals for EWBG are strongly constrained by precision Higgs measurements and searches for new sources of $C P$ violation. In this study, we have focused on a realization of EWBG in which baryon creation occurs in an initial phase transition to an exotic vacuum exhibiting broken electroweak symmetry [31]. A later transition to a Standard Model-like Higgs vacuum then occurs at some lower temperature. Naively, this allows for the new physics involved in strengthening the electroweak phase transition to be decoupled from the properties of the SM-like Higgs boson observed at the LHC. The results of our study suggest that this is not entirely true.

While two-stage electroweak phase transitions have been studied previously and applied to EWBG, our investigation is novel in several respects. We have presented a simplified model that makes clear the conditions required for two-stage electroweak symmetry breaking in general extensions of the SM Higgs sector. We used the intuition gained from this toy model to study two-stage phase transitions in inert two-Higgs-doublet models, with and without additional matter content. These scenarios can exhibit two-step phase transitions suitable for EWBG. Relative to the triplet model studied in Ref. [31, the inert doublet model appears to have a larger parameter region where this can occur, and it avoids the possibility of additional baryon washout by monopoles after the first transition.

A seemingly general result of our study is that two-stage electroweak phase transitions predict new light electroweakly-charged particles. In the portion of the inert doublet model parameter space consistent with two-stage EWBG, these new states are so light that they are ruled by direct collider searches. We illustrated a generic solution to this problem in the form of a singlet-induced mass term for the problematic states that can still allow for successful electroweak baryogenesis from a two-stage transition. Although the singlet contribution to the sterile particle masses allows for two-stage scenarios consistent with current experimental data, the models we have considered here still generically feature potentially observable deviations in the rate of $h \rightarrow \gamma \gamma$ relative to the Standard Model due to loop contributions from the new exotic states. This prediction seems difficult to avoid since increasing the singlet-inert mixed quartic coupling to push up the electroweak-preserving masses of these states would also lead to larger thermal mass corrections to the exotic multiplet. For large 
couplings, the phase transition to the SM-like Higgs vacuum will then occur first. We expect this feature to be generic, holding beyond just the inert doublet (and triplet) scenarios considered here. This exemplifies the virtue of electroweak baryogenesis as a concretely testable scenario and one that deserves to be studied thoroughly, both theoretically and in experiments exploring the electroweak scale.

\section{Acknowledgements}

The authors would like to thank Wei Chao, Grigory Ovanesyan, Michael Ramsey-Musolf, Brian Shuve, and Peter Winslow for useful discussions. We also thank Michael RamseyMusolf for comments on this manuscript. This research is supported by the National Science and Engineering Research Council of Canada (NSERC). Research at the Perimeter Institute is supported in part by the Government of Canada through Industry Canada, and by the Province of Ontario through the Ministry of Research and Information (MRI). NB thanks UC Santa Cruz and the Santa Cruz Institute for Particle Physics for hospitality where part of this work was completed. DM thanks the Perimeter Institute for their hospitality during part of the completion of this work. CT acknowledges support of the Spanish Government through grant FPA2011-24568 (MICINN), and thanks TRIUMF, where the ideas for this work were first discussed.

\section{A One-Loop Beta Functions and Anomalous Dimensions}

In this appendix we reproduce the beta functions and anomalous dimensions relevant for computing the renormalization group-improved effective potential. We follow the standard convention of defining the beta function of a coupling $\kappa$ to be

$$
\beta_{\kappa}=\mu \frac{d \kappa}{d \mu}
$$

where $\mu$ is the renormalization scale. For a field $\varphi$ with an associated (unmixed) wavefunction renormalization factor $Z_{\varphi}$ we define the anomalous dimension to be

$$
\gamma_{\varphi}=\frac{1}{2 Z_{\varphi}} \mu \frac{d Z_{\varphi}}{d \mu} .
$$

We computed these at two loops in the $\overline{\mathrm{MS}}$ scheme in dimensional regularization following the general results of Refs. [98 101]. In the formulae below, we only show the one-loop result

for compactness. Note as well that we use the GUT normalization for the hypercharge gauge coupling, $g_{1}=\sqrt{5 / 3} g^{\prime}$. 
The beta functions for the inert doublet plus singlet model defined in Section 4.6, in the enhanced-symmetry limit $\lambda_{5}=0$, are

$$
\begin{aligned}
& \beta_{g_{1}}=\beta_{g_{1}}^{\mathrm{SM}}+\frac{1}{(4 \pi)^{2}} \frac{g_{1}^{3}}{10} \\
& \beta_{g_{2}}=\beta_{g_{2}}^{\mathrm{SM}}+\frac{1}{(4 \pi)^{2}} \frac{g_{2}^{3}}{6} \\
& \beta_{g_{3}}=\beta_{g_{3}}^{\mathrm{SM}} \\
& \beta_{\lambda}=\beta_{\lambda_{1}}^{\mathrm{SM}}+\frac{1}{(4 \pi)^{2}}\left[4 \lambda_{\Phi H} \tilde{\lambda}_{\Phi H}+2 \tilde{\lambda}_{\Phi H}^{2}+\lambda_{S H}^{2}+4 \lambda_{\Phi H}^{2}\right], \\
& \beta_{m_{H}^{2}}=\beta_{m_{H}^{2}}^{\mathrm{SM}}+\frac{1}{(4 \pi)^{2}}\left[m_{\Phi}^{2}\left(2 \tilde{\lambda}_{\Phi H}+4 \lambda_{\Phi H}\right)+\lambda_{S H} m_{S}^{2}\right] \\
& \beta_{\lambda_{\Phi}}=\frac{1}{(4 \pi)^{2}}\left[2 \tilde{\lambda}_{\Phi H}^{2}+4 \lambda_{\Phi H} \tilde{\lambda}_{\Phi H}+\left(-\frac{9}{5} g_{1}^{2}-9 g_{2}^{2}\right) \lambda_{\Phi}+\frac{27 g_{1}^{4}}{100}+\frac{9}{10} g_{2}^{2} g_{1}^{2}+\frac{9 g_{2}^{4}}{4}+12 \lambda_{\Phi}^{2}\right. \\
& \left.+4 \lambda_{\Phi H}^{2}+\lambda_{S \Phi}^{2}\right] \\
& \beta_{m_{\Phi}^{2}}=\frac{1}{(4 \pi)^{2}}\left[m_{H}^{2}\left(2 \tilde{\lambda}_{\Phi H}+4 \lambda_{\Phi H}\right)+m_{\Phi}^{2}\left(-\frac{9}{10} g_{1}^{2}-\frac{9 g_{2}^{2}}{2}+6 \lambda_{\Phi}\right)+m_{S}^{2} \lambda_{S \Phi}\right] \\
& \beta_{\lambda_{\Phi H}}=\frac{1}{(4 \pi)^{2}}\left[\lambda_{\Phi}\left(2 \tilde{\lambda}_{\Phi H}+6 \lambda_{\Phi H}\right)+2 \tilde{\lambda}_{\Phi H}^{2}+\lambda\left(2 \tilde{\lambda}_{\Phi H}+6 \lambda_{\Phi H}\right)+\lambda_{\Phi H}\left(6 y_{b}^{2}-\frac{9}{5} g_{1}^{2}-9 g_{2}^{2}\right.\right. \\
& \left.\left.+6 y_{t}^{2}+2 y_{\tau}^{2}\right)+\frac{27 g_{1}^{4}}{100}-\frac{9}{10} g_{2}^{2} g_{1}^{2}+\frac{9 g_{2}^{4}}{4}+4 \lambda_{\Phi H}^{2}+\lambda_{S H} \lambda_{S \Phi}\right] \\
& \beta_{\tilde{\lambda}_{\Phi H}}=\frac{1}{(4 \pi)^{2}}\left[\tilde{\lambda}_{\Phi H}\left(6 y_{b}^{2}-\frac{9}{5} g_{1}^{2}-9 g_{2}^{2}+8 \lambda_{\Phi H}+6 y_{t}^{2}+2 y_{\tau}^{2}\right)+2 \lambda_{\Phi} \tilde{\lambda}_{\Phi H}+4 \tilde{\lambda}_{\Phi H}^{2}+2 \lambda \tilde{\lambda}_{\Phi H}\right. \\
& \left.+\frac{9}{5} g_{1}^{2} g_{2}^{2}\right] \\
& \beta_{\lambda_{S}}=\frac{1}{(4 \pi)^{2}}\left[3 \lambda_{S}^{2}+12 \lambda_{S H}^{2}+12 \lambda_{S \Phi}^{2}\right] \\
& \beta_{m_{S}^{2}}=\frac{1}{(4 \pi)^{2}}\left[4 m_{H}^{2} \lambda_{S H}+\lambda_{S} m_{S}^{2}+4 m_{\Phi}^{2} \lambda_{S \Phi}\right] \\
& \beta_{\lambda_{S H}}=\frac{1}{(4 \pi)^{2}}\left[\lambda_{S H}\left(6 y_{b}^{2}-\frac{9}{10} g_{1}^{2}-\frac{9 g_{2}^{2}}{2}+6 y_{t}^{2}+2 y_{\tau}^{2}\right)+\lambda_{S} \lambda_{S H}+4 \lambda_{S H}^{2}+6 \lambda \lambda_{S H}\right. \\
& \left.+2 \lambda_{S \Phi} \tilde{\lambda}_{\Phi H}+4 \lambda_{\Phi H} \lambda_{S \Phi}\right] \\
& \beta_{\lambda_{S \Phi}}=\frac{1}{(4 \pi)^{2}}\left[2 \lambda_{S H} \tilde{\lambda}_{\Phi H}+\lambda_{S \Phi}\left(-\frac{9}{10} g_{1}^{2}-\frac{9 g_{2}^{2}}{2}+6 \lambda_{\Phi}+\lambda_{S}\right)+4 \lambda_{\Phi H} \lambda_{S H}+4 \lambda_{S \Phi}^{2}\right] .
\end{aligned}
$$

In the equations above, the $\beta_{\delta}^{\mathrm{SM}}$ denotes the beta function of the corresponding coupling in the Standard Model. The above beta functions may also be applied to the pure inert doublet 
model by setting $\lambda_{S}=\lambda_{S H}=\lambda_{S \Phi}=0=m_{S}^{2}$. The anomalous dimensions are

$$
\begin{aligned}
\gamma_{H} & =\beta_{\gamma_{H}}^{\mathrm{SM}}, \\
\gamma_{\Phi} & =\frac{1}{(4 \pi)^{2}}\left[-\frac{9}{20} g_{1}^{2}-\frac{9 g_{2}^{2}}{4}\right], \\
\gamma_{S} & =0 .
\end{aligned}
$$

\section{B One-Loop Thermal Masses}

The leading high-temperature contributions to the bosonic self-energies are needed in order to perform a daisy resummation of the corrections due to the bosonic $n=0$ Matsubara modes. These have been derived in the SM in Ref. [102, while partial results for inert doublet models are given in Refs. [55, 103]. We extend these results to include an additional singlet as in Section 4.6 .

For the gauge fields, only the self-energies corresponding to longitudinal polarizations receive thermal corrections at leading order. Denoting these with $\Pi_{L}^{W}$ and $\Pi_{L}^{B}$ for the $S U(2)_{L}$ and $U(1)_{Y}$ gauge bosons, respectively, one has

$$
\Pi_{L}^{W}=2 g_{2}^{2} T^{2}, \quad \Pi_{L}^{B}=\frac{6}{5} g_{1}^{2} T^{2} .
$$

Taking these together with the zero-temperature results, the temperature-corrected mass eigenvalues for the longitudinal components of the gauge bosons in a general $(h, \phi) \neq(0,0)$ background are

$$
\begin{aligned}
m_{L}^{W} & =\frac{1}{4} g_{2}^{2}\left(h^{2}+\phi^{2}\right)+2 g_{2}^{2} T^{2}, \\
m_{L}^{Z} & =\frac{1}{8}\left(g_{2}^{2}+\frac{3}{5} g_{1}^{2}\right)\left(h^{2}+\phi^{2}\right)+g_{2}^{2} T^{2}+\frac{3}{5} g_{1}^{2} T^{2}+\Delta_{1}, \\
m_{L}^{\gamma} & =\frac{1}{8}\left(g_{2}^{2}+\frac{3}{5} g_{1}^{2}\right)\left(h^{2}+\phi^{2}\right)+g_{2}^{2} T^{2}+\frac{3}{5} g_{1}^{2} T^{2}-\Delta_{1}, \\
\Delta_{1} & =\frac{1}{40} \sqrt{\left(5 g_{2}^{2}+3 g_{1}^{2}\right)^{2}\left(\phi^{2}+8 T^{2}+h^{2}\right)^{2}-960 g_{2}^{2} g_{1}^{2} T^{2}\left(\phi^{2}+4 T^{2}+h^{2}\right)} .
\end{aligned}
$$

The leading thermal self-energies for the scalar doublets and the singlet are

$$
\begin{aligned}
\Pi_{H^{\dagger} H} & =\left(\frac{1}{4} \lambda+\frac{1}{6} \lambda_{\Phi H}+\frac{1}{12} \tilde{\lambda}_{\Phi H}+\frac{1}{24} \lambda_{S H}+\frac{1}{4} y_{t}^{2}+\frac{3}{16} g_{2}^{2}+\frac{3}{80} g_{1}^{2}\right) T^{2}, \\
\Pi_{\Phi^{\dagger} \Phi} & =\left(\frac{1}{4} \lambda_{\Phi}+\frac{1}{6} \lambda_{\Phi H}+\frac{1}{12} \tilde{\lambda}_{\Phi H}+\frac{1}{24} \lambda_{S \Phi}+\frac{3}{16} g_{2}^{2}+\frac{3}{80} g_{1}^{2}\right) T^{2}, \\
\Pi_{S S} & =\left(\frac{1}{24} \lambda_{S}+\frac{1}{6} \lambda_{S H}+\frac{1}{6} \lambda_{S \Phi}\right) T^{2} .
\end{aligned}
$$


As above, these can be applied to the pure inert doublet model (without the singlet) by setting $\lambda_{S H}=\lambda_{S \Phi}=\lambda_{S}=0$.

\section{References}

[1] M. Trodden, Electroweak baryogenesis, Rev.Mod.Phys. 71 (1999) 1463-1500, hep-ph/9803479.

[2] A. G. Cohen, D. Kaplan, and A. Nelson, Progress in electroweak baryogenesis, Ann.Rev.Nucl.Part.Sci. 43 (1993) 27-70, hep-ph/9302210].

[3] D. E. Morrissey and M. J. Ramsey-Musolf, Electroweak baryogenesis, New J.Phys. 14 (2012) 125003, arXiv:1206.2942.

[4] A. Sakharov, Violation of CP Invariance, C Asymmetry, and Baryon Asymmetry of the Universe, Pisma Zh.Eksp.Teor.Fiz. 5 (1967) 32-35.

[5] H. H. Patel and M. J. Ramsey-Musolf, Baryon Washout, Electroweak Phase Transition, and Perturbation Theory, JHEP 1107 (2011) 029, arXiv:1101.4665.

[6] M. Garny and T. Konstandin, On the gauge dependence of vacuum transitions at finite temperature, JHEP 1207 (2012) 189, arXiv:1205.3392.

[7] K. Kajantie, M. Laine, K. Rummukainen, and M. E. Shaposhnikov, The Electroweak phase transition: A Nonperturbative analysis, Nucl.Phys. B466 (1996) 189-258, hep-lat/9510020.

[8] K. Kajantie, M. Laine, K. Rummukainen, and M. E. Shaposhnikov, Is there a hot electroweak phase transition at $m(H)$ larger or equal to $m(W)$ ?, Phys.Rev.Lett. 77 (1996) 2887-2890, hep-ph/9605288.

[9] M. Gavela, P. Hernandez, J. Orloff, and O. Pene, Standard model CP violation and baryon asymmetry, Mod.Phys.Lett. A9 (1994) 795-810, hep-ph/9312215.

[10] P. Huet and E. Sather, Electroweak baryogenesis and standard model CP violation, Phys.Rev. D51 (1995) 379-394, hep-ph/9404302.

[11] M. Gavela, P. Hernandez, J. Orloff, O. Pene, and C. Quimbay, Standard model CP violation and baryon asymmetry. Part 2: Finite temperature, Nucl.Phys. B430 (1994) 382-426, hep-ph/9406289.

[12] M. Carena, M. Quiros, and C. Wagner, Opening the window for electroweak baryogenesis, Phys.Lett. B380 (1996) 81-91, hep-ph/9603420|. 
[13] D. Delepine, J. Gerard, R. Gonzalez Felipe, and J. Weyers, A Light stop and electroweak baryogenesis, Phys.Lett. B386 (1996) 183-188, [hep-ph/9604440].

[14] A. Davies, C. Froggatt, and R. Moorhouse, Electroweak baryogenesis in the next-to-minimal supersymmetric model, Phys.Lett. B372 (1996) 88-94, hep-ph/9603388].

[15] S. Huber and M. Schmidt, Electroweak baryogenesis: Concrete in a SUSY model with a gauge singlet, Nucl.Phys. B606 (2001) 183-230, [hep-ph/0003122].

[16] A. Menon, D. Morrissey, and C. Wagner, Electroweak baryogenesis and dark matter in the nMSSM, Phys.Rev. D70 (2004) 035005, [hep-ph/0404184].

[17] A. Menon and D. E. Morrissey, Higgs Boson Signatures of MSSM Electroweak Baryogenesis, Phys.Rev. D79 (2009) 115020, arXiv:0903.3038.

[18] T. Cohen, D. E. Morrissey, and A. Pierce, Electroweak Baryogenesis and Higgs Signatures, Phys.Rev. D86 (2012) 013009, arXiv:1203.2924.

[19] D. Curtin, P. Jaiswal, and P. Meade, Excluding Electroweak Baryogenesis in the MSSM, JHEP 1208 (2012) 005, arXiv:1203.2932].

[20] M. Carena, G. Nardini, M. Quiros, and C. E. Wagner, MSSM Electroweak Baryogenesis and LHC Data, JHEP 1302 (2013) 001, arXiv:1207.6330.

[21] K. Krizka, A. Kumar, and D. E. Morrissey, Very Light Scalar Top Quarks at the LHC, Phys.Rev. D87 (2013), no. 9 095016, arXiv:1212.4856.

[22] A. Delgado, G. F. Giudice, G. Isidori, M. Pierini, and A. Strumia, The light stop window, Eur.Phys.J. C73 (2013), no. 3 2370, arXiv:1212.6847.

[23] W. Huang, Z. Kang, J. Shu, P. Wu, and J. M. Yang, New insights in the electroweak phase transition in the NMSSM, Phys.Rev. D91 (2015), no. 2025006 , arXiv:1405.1152.

[24] J. Kozaczuk, S. Profumo, L. S. Haskins, and C. L. Wainwright, Cosmological Phase Transitions and their Properties in the NMSSM, JHEP 1501 (2015) 144, arXiv:1407.4134.

[25] J. Espinosa, T. Konstandin, J. No, and M. Quiros, Some Cosmological Implications of Hidden Sectors, Phys.Rev. D78 (2008) 123528, |arXiv:0809.3215|.

[26] C. Tamarit, Higgs vacua with potential barriers, Phys.Rev. D90 (2014), no. 5055024 , arXiv:1404.7673.

[27] D. J. Chung, A. J. Long, and L.-T. Wang, 125 GeV Higgs boson and electroweak phase transition model classes, Phys.Rev. D87 (2013), no. 2 023509, arXiv:1209.1819. 
[28] M. Pospelov and A. Ritz, Electric dipole moments as probes of new physics, Annals Phys. 318 (2005) 119-169, hep-ph/0504231.

[29] V. Cirigliano, Y. Li, S. Profumo, and M. J. Ramsey-Musolf, MSSM Baryogenesis and Electric Dipole Moments: An Update on the Phenomenology, JHEP 1001 (2010) 002, arXiv:0910.4589.

[30] J. Shu and Y. Zhang, Impact of a CP Violating Higgs Sector: From LHC to Baryogenesis, Phys.Rev.Lett. 111 (2013), no. 9 091801, arXiv:1304.0773.

[31] H. H. Patel and M. J. Ramsey-Musolf, Stepping Into Electroweak Symmetry Breaking: Phase Transitions and Higgs Phenomenology, Phys.Rev. D88 (2013) 035013, arXiv: 1212.5652.

[32] A. Ahriche, What is the criterion for a strong first order electroweak phase transition in singlet models?, Phys.Rev. D75 (2007) 083522, hep-ph/0701192.

[33] S. Profumo, M. J. Ramsey-Musolf, and G. Shaughnessy, Singlet Higgs phenomenology and the electroweak phase transition, JHEP 0708 (2007) 010, arXiv:0705.2425.

[34] D. Curtin, P. Meade, and C.-T. Yu, Testing Electroweak Baryogenesis with Future Colliders, JHEP 1411 (2014) 127, arXiv: 1409.0005.

[35] D. Land and E. D. Carlson, Two stage phase transition in two Higgs models, Phys.Lett. B292 (1992) 107-112, hep-ph/9208227.

[36] A. Hammerschmitt, J. Kripfganz, and M. Schmidt, Baryon asymmetry from a two stage electroweak phase transition?, Z.Phys. C64 (1994) 105-110, hep-ph/9404272].

[37] R. Enberg, J. Rathsman, and G. Wouda, Higgs properties in a broken Inert Doublet Model, JHEP 1308 (2013) 079, arXiv:1304.1714].

[38] R. Enberg, J. Rathsman, and G. Wouda, Higgs phenomenology in the Stealth Doublet Model, arXiv:1311.4367.

[39] P. B. Arnold and L. D. McLerran, Sphalerons, Small Fluctuations and Baryon Number Violation in Electroweak Theory, Phys.Rev. D36 (1987) 581.

[40] M. Quiros, Finite temperature field theory and phase transitions, hep-ph/9901312.

[41] A. Ahriche, T. A. Chowdhury, and S. Nasri, Sphalerons and the Electroweak Phase Transition in Models with Higher Scalar Representations, JHEP 1411 (2014) 096, arXiv:1409.4086.

[42] T. Hambye and M. H. Tytgat, Electroweak symmetry breaking induced by dark matter, Phys.Lett. B659 (2008) 651-655, arXiv:0707.0633. 
[43] ATLAS Collaboration, G. Aad et al., Measurement of the Higgs boson mass from the $H \rightarrow \gamma \gamma$ and $H \rightarrow Z Z^{*} \rightarrow 4 \ell$ channels with the ATLAS detector using $25 \mathrm{fb}^{-1}$ of pp collision data, Phys.Rev. D90 (2014), no. 5 052004, arXiv:1406.3827.

[44] CMS Collaboration, V. Khachatryan et al., Precise determination of the mass of the Higgs boson and tests of compatibility of its couplings with the standard model predictions using proton collisions at 7 and $8 \mathrm{TeV}$, arXiv:1412.8662.

[45] ATLAS, CMS Collaboration, G. Aad et al., Combined Measurement of the Higgs Boson Mass in pp Collisions at $\sqrt{s}=7$ and 8 TeV with the ATLAS and CMS Experiments, arXiv:1503.07589.

[46] S. P. Martin, Two loop scalar self energies in a general renormalizable theory at leading order in gauge couplings, Phys.Rev. D70 (2004) 016005, hep-ph/0312092.

[47] S. P. Martin, Taming the Goldstone contributions to the effective potential, Phys.Rev. D90 (2014), no. 1 016013, arXiv:1406.2355.

[48] J. Elias-Miro, J. Espinosa, and T. Konstandin, Taming Infrared Divergences in the Effective Potential, JHEP 1408 (2014) 034, arXiv:1406.2652].

[49] R. R. Parwani, Resummation in a hot scalar field theory, Phys.Rev. D45 (1992) 4695, hep-ph/9204216.

[50] P. B. Arnold and O. Espinosa, The Effective potential and first order phase transitions: Beyond leading-order, Phys.Rev. D47 (1993) 3546, hep-ph/9212235.

[51] C. L. Wainwright, CosmoTransitions: Computing Cosmological Phase Transition Temperatures and Bubble Profiles with Multiple Fields, Comput.Phys.Commun. 183 (2012) 2006-2013, arXiv:1109.4189|.

[52] G. W. Anderson and L. J. Hall, The Electroweak phase transition and baryogenesis, Phys.Rev. D45 (1992) 2685-2698.

[53] I. Ginzburg, K. Kanishev, M. Krawczyk, and D. Sokolowska, Evolution of Universe to the present inert phase, Phys.Rev. D82 (2010) 123533, arXiv:1009.4593].

[54] M. Jarvinen, T. A. Ryttov, and F. Sannino, Extra Electroweak Phase Transitions from Strong Dynamics, Phys.Lett. B680 (2009) 251-254, arXiv:0901.0496.

[55] G. Gil, P. Chankowski, and M. Krawczyk, Inert Dark Matter and Strong Electroweak Phase Transition, Phys.Lett. B717 (2012) 396-402, [arXiv:1207.0084].

[56] T. A. Chowdhury, M. Nemevsek, G. Senjanovic, and Y. Zhang, Dark Matter as the Trigger of Strong Electroweak Phase Transition, JCAP 1202 (2012) 029, arXiv:1110.5334. 
[57] D. Borah and J. M. Cline, Inert Doublet Dark Matter with Strong Electroweak Phase Transition, Phys.Rev. D86 (2012) 055001, arXiv:1204.4722.

[58] J. M. Cline and K. Kainulainen, Improved Electroweak Phase Transition with Subdominant Inert Doublet Dark Matter, Phys.Rev. D87 (2013), no. 7 071701, arXiv:1302.2614.

[59] S. S. AbdusSalam and T. A. Chowdhury, Scalar Representations in the Light of Electroweak Phase Transition and Cold Dark Matter Phenomenology, JCAP 1405 (2014) 026, arXiv:1310.8152.

[60] M. E. Peskin and T. Takeuchi, A New constraint on a strongly interacting Higgs sector, Phys.Rev.Lett. 65 (1990) 964-967.

[61] M. E. Peskin and T. Takeuchi, Estimation of oblique electroweak corrections, Phys.Rev. D46 (1992) 381-409.

[62] R. Barbieri, L. J. Hall, and V. S. Rychkov, Improved naturalness with a heavy Higgs: An Alternative road to LHC physics, Phys.Rev. D74 (2006) 015007, hep-ph/0603188.

[63] Q.-H. Cao, E. Ma, and G. Rajasekaran, Observing the Dark Scalar Doublet and its Impact on the Standard-Model Higgs Boson at Colliders, Phys.Rev. D76 (2007) 095011, arXiv:0708.2939.

[64] E. M. Dolle and S. Su, The Inert Dark Matter, Phys.Rev. D80 (2009) 055012, arXiv:0906.1609.

[65] Particle Data Group Collaboration, K. Olive et al., Review of Particle Physics, Chin.Phys. C38 (2014) 090001.

[66] OPAL Collaboration, G. Abbiendi et al., Search for anomalous production of dilepton events with missing transverse momentum in $e^{+} e^{-}$collisions at $\sqrt{s}=183$ GeV to 209 GeV, Eur.Phys.J. C32 (2004) 453-473, hep-ex/0309014].

[67] OPAL Collaboration, G. Abbiendi et al., Search for chargino and neutralino production at $\sqrt{s}=192 \mathrm{GeV}$ to $209 \mathrm{GeV}$ at LEP, Eur.Phys.J. C35 (2004) 1-20, hep-ex/0401026.

[68] A. Pierce and J. Thaler, Natural Dark Matter from an Unnatural Higgs Boson and New Colored Particles at the TeV Scale, JHEP 0708 (2007) 026, hep-ph/0703056.

[69] E. Lundstrom, M. Gustafsson, and J. Edsjo, The Inert Doublet Model and LEP II Limits, Phys.Rev. D79 (2009) 035013, arXiv:0810.3924.

[70] E. Dolle, X. Miao, S. Su, and B. Thomas, Dilepton Signals in the Inert Doublet Model, Phys.Rev. D81 (2010) 035003, arXiv:0909.3094. 
[71] X. Miao, S. Su, and B. Thomas, Trilepton Signals in the Inert Doublet Model, Phys.Rev. D82 (2010) 035009, arXiv:1005.0090].

[72] M. Gustafsson, S. Rydbeck, L. Lopez-Honorez, and E. Lundstrom, Status of the Inert Doublet Model and the Role of multileptons at the LHC, Phys.Rev. D86 (2012) 075019, arXiv: 1206.6316.

[73] G. Belanger, B. Dumont, A. Goudelis, B. Herrmann, S. Kraml, et al., Dilepton constraints in the Inert Doublet Model from Run 1 of the LHC, arXiv:1503.07367.

[74] A. Arhrib, Y.-L. S. Tsai, Q. Yuan, and T.-C. Yuan, An Updated Analysis of Inert Higgs Doublet Model in light of the Recent Results from LUX, PLANCK, AMS-02 and LHC, JCAP 1406 (2014) 030, arXiv:1310.0358.

[75] G. Belanger, B. Dumont, U. Ellwanger, J. Gunion, and S. Kraml, Global fit to Higgs signal strengths and couplings and implications for extended Higgs sectors, Phys.Rev. D88 (2013) 075008, arXiv:1306.2941.

[76] B. Swiezewska and M. Krawczyk, Diphoton rate in the inert doublet model with a 125 GeV Higgs boson, Phys.Rev. D88 (2013), no. 3 035019, arXiv:1212.4100.

[77] A. Arhrib, R. Benbrik, and N. Gaur, $H \rightarrow \gamma \gamma$ in Inert Higgs Doublet Model, Phys.Rev. D85 (2012) 095021, arXiv:1201.2644.

[78] A. Celis, V. Ilisie, and A. Pich, LHC constraints on two-Higgs doublet models, JHEP 1307 (2013) 053, arXiv:1302.4022.

[79] G. K. John F. Gunion, Howard E. Haber and S. Dawson, The Higgs Hunter's Guide. Perseus Publishing, 1990.

[80] G. Branco, P. Ferreira, L. Lavoura, M. Rebelo, M. Sher, et al., Theory and phenomenology of two-Higgs-doublet models, Phys.Rept. 516 (2012) 1-102, arXiv:1106.0034.

[81] A. Djouadi, The Anatomy of electro-weak symmetry breaking. II. The Higgs bosons in the minimal supersymmetric model, Phys.Rept. 459 (2008) 1-241, hep-ph/0503173].

[82] M. Spira, A. Djouadi, D. Graudenz, and P. Zerwas, Higgs boson production at the LHC, Nucl.Phys. B453 (1995) 17-82, hep-ph/9504378.

[83] A. Djouadi, J. Kalinowski, and M. Spira, HDECAY: A Program for Higgs boson decays in the standard model and its supersymmetric extension, Comput.Phys.Commun. 108 (1998) 56-74, hep-ph/9704448].

[84] ATLAS Collaboration, G. Aad et al., Measurement of Higgs boson production in the diphoton decay channel in pp collisions at center-of-mass energies of 7 and $8 \mathrm{TeV}$ with the ATLAS detector, arXiv:1408.7084. 
[85] CMS Collaboration, V. Khachatryan et al., Observation of the diphoton decay of the Higgs boson and measurement of its properties, Eur.Phys.J. C74 (2014), no. 10 3076, arXiv:1407.0558.

[86] L. Lopez Honorez, E. Nezri, J. F. Oliver, and M. H. Tytgat, The Inert Doublet Model: An Archetype for Dark Matter, JCAP 0702 (2007) 028, hep-ph/0612275].

[87] A. Goudelis, B. Herrmann, and O. Stål, Dark matter in the Inert Doublet Model after the discovery of a Higgs-like boson at the LHC, JHEP 1309 (2013) 106, arXiv:1303.3010.

[88] N. Blinov, S. Profumo, and T. Stefaniak, The electroweak phase transition in the inert doublet model, In Preparation (2015).

[89] C. Arina, F.-S. Ling, and M. H. Tytgat, IDM and iDM or The Inert Doublet Model and Inelastic Dark Matter, JCAP 0910 (2009) 018, arXiv:0907.0430].

[90] XENON100 Collaboration, E. Aprile et al., Dark Matter Results from 225 Live Days of XENON100 Data, Phys.Rev.Lett. 109 (2012) 181301, arXiv:1207.5988.

[91] LUX Collaboration, D. Akerib et al., First results from the LUX dark matter experiment at the Sanford Underground Research Facility, Phys.Rev.Lett. 112 (2014) 091303, arXiv:1310.8214.

[92] R. Schabinger and J. D. Wells, A Minimal spontaneously broken hidden sector and its impact on Higgs boson physics at the large hadron collider, Phys.Rev. D72 (2005) 093007, hep-ph/0509209.

[93] B. Patt and F. Wilczek, Higgs-field portal into hidden sectors, hep-ph/0605188.

[94] B. Batell, M. Pospelov, and A. Ritz, Exploring Portals to a Hidden Sector Through Fixed Targets, Phys.Rev. D80 (2009) 095024, arXiv:0906.5614].

[95] J. D. Clarke, R. Foot, and R. R. Volkas, Phenomenology of a very light scalar $\left(100 \mathrm{MeV}<m_{h}<10 \mathrm{GeV}\right)$ mixing with the SM Higgs, JHEP 1402 (2014) 123, arXiv:1310.8042.

[96] A. Falkowski, C. Gross, and O. Lebedev, A second Higgs from the Higgs portal, arXiv: 1502.01361.

[97] T. Kibble, Some Implications of a Cosmological Phase Transition, Phys.Rept. 67 (1980) 183.

[98] M. E. Machacek and M. T. Vaughn, Two Loop Renormalization Group Equations in a General Quantum Field Theory. 1. Wave Function Renormalization, Nucl.Phys. B222 (1983) 83. 
[99] M. E. Machacek and M. T. Vaughn, Two Loop Renormalization Group Equations in a General Quantum Field Theory. 2. Yukawa Couplings, Nucl.Phys. B236 (1984) 221.

[100] M. E. Machacek and M. T. Vaughn, Two Loop Renormalization Group Equations in a General Quantum Field Theory. 3. Scalar Quartic Couplings, Nucl.Phys. B249 (1985) 70.

[101] M.-x. Luo, H.-w. Wang, and Y. Xiao, Two loop renormalization group equations in general gauge field theories, Phys.Rev. D67 (2003) 065019, hep-ph/0211440.

[102] M. Carrington, The Effective potential at finite temperature in the Standard Model, Phys.Rev. D45 (1992) 2933-2944.

[103] J. M. Cline, K. Kainulainen, and A. P. Vischer, Dynamics of two Higgs doublet CP violation and baryogenesis at the electroweak phase transition, Phys.Rev. D54 (1996) 2451-2472, hep-ph/9506284. 